\title{
FDR, an easier way to NNLO calculations: a two-loop case study
}

\author{
Alice Maria Donati ${ }^{\mathrm{a}}$, Roberto Pittau ${ }^{\mathrm{b}}$ \\ Departamento de Física Teórica y del Cosmos and CAFPE, Universidad de Granada, Campus Fuentenueva s. n., 18071 Granada, Spain
}

Received: 13 March 2014 / Accepted: 14 April 2014 / Published online: 3 May 2014

(C) The Author(s) 2014. This article is published with open access at Springerlink.com

\begin{abstract}
In this paper we illustrate the simplifications produced by FDR in NNLO computations. We show with an explicit example that—due to its four-dimensionality-FDR does not require an order-by-order renormalization and that, unlike the one-loop case, FDR and dimensional regularization generate intermediate two-loop results which are no longer linked by a simple subtraction of the ultraviolet (UV) poles in $\epsilon$. Our case study is the two-loop amplitude for $H \rightarrow \gamma \gamma$, mediated by an infinitely heavy top loop, in the presence of gluonic corrections. We use this to elucidate how gauge invariance is preserved with no need of introducing counterterms in the Lagrangian. In addition, we discuss a possible four-dimensional approach to the infrared problem compatible with the FDR treatment of the UV infinities.
\end{abstract}

\section{Introduction}

Computing radiative corrections has become of uppermost importance in particle phenomenology [1]. The present lack of unexpected signals at the LHC pulls the effects of New Physics in a domain where small discrepancies have to be searched via detailed comparisons between experimental results and precise calculations of the Standard Model background. Due to the large QCD coupling constant, precise predictions at the LHC often require NNLO accuracy. On the other hand, calculations based on two or more loops in the complete Electroweak (EW) model will be mandatory at the future International Linear Collider to meet the experimental accuracy foreseen, for example, in Higgs Physics [2].

While NLO techniques are very well established [3-8], [9-15], work is ongoing to solve the NNLO problem in its full generality [16]. As for the virtual sector, progress has been recently achieved by extending generalized unitarity techniques at two loops [17-21], while the antenna

\footnotetext{
a e-mail: adonati@ugr.es

b e-mail: pittau@ugr.es
}

subtraction [22,23] and sector decomposition [24-27] methods look promising tools to deal with IR divergences beyond NLO [28].

In this paper we investigate the possibility of further simplifying NNLO computations by abandoning dimensional regularization [29]. Despite its known virtues, DR requires a heavy analytic work aimed at subtracting powers of $1 / \epsilon$ of UV or IR origin even before attacking the calculation of the finite physical part. For instance, DR forces an orderby-order iterative renormalization, which is especially cumbersome when computing loop corrections in the EW model or in SUSY: the full set of one-loop counterterms has to be determined and added in a two-loop computation, and so on. Furthermore, loop functions used at a given perturbative level must be further expanded in $\epsilon$-when appearing at higher orders - to include terms generating $\mathcal{O}\left(\epsilon^{0}\right)$ contributions when multiplied by the new poles. Such complications arise in DR because constants needed to preserve the symmetries of the Lagrangian are often produced by $\epsilon \epsilon$ terms, which are kept under control by the iterative renormalization.

This has driven us to study the performances of FDR [30] as a simpler four-dimensional approach beyond one loop. ${ }^{1}$ The key point of FDR is that the use of counterterms is avoided by defining a four-dimensional and UV-free loop integration in a way compatible with shift and gauge invariance. Having done this, the correct results automatically emerge once the theory is fixed in terms of physical observables by means of a finite renormalization relating the parameters of the Lagrangian to measured quantities. In addition, IR infinities can be naturally accommodated within the same four-dimensional framework used to cope with the UV divergences. This is why we envisage in FDR a great potential to reduce the complexity of the NNLO calculations, especially when used together with numerical techniques.

In this paper we present, as the first example of a twoloop FDR calculation, the QCD corrections to the top-

\footnotetext{
${ }^{1}$ Other four-dimensional treatments are listed in [31-36].
} 
loop-mediated Higgs decay into two photons, in the limit $m_{\text {top }} \rightarrow \infty$. This computation gives the opportunity to fully appreciate the simplifications due to the four-dimensionality of the approach in a realistic two-loop case study. ${ }^{2}$ In the next section we review the general FDR idea with special emphasis on the two-loop case. We discuss, in particular, the shift and gauge invariance properties of the FDR integration, the main differences with DR, and the IR problem. The two-loop FDR calculation of $H \rightarrow \gamma \gamma$ is presented in Sect. 3 and the technical details are collected in the final appendices.

\section{FDR and the importance of working in four dimensions}

\subsection{Definition of the FDR loop integral}

FDR subtracts UV divergences at the integrand level. This is obtained in two steps. Firstly, the propagators of the particles flowing in the loops are given a common additional term $-\mu^{2}$, formally identified with the $+i 0$ propagator prescription. For example, vector-boson and fermion propagators with momentum $\left(q+p_{i}\right)$ and mass $M_{i}$ read, ${ }^{3}$ in the unitary gauge,

$\frac{g^{\alpha \beta}-\left(q+p_{i}\right)^{\alpha}\left(q+p_{i}\right)^{\beta} / M_{i}^{2}}{\bar{D}_{p_{i}}} \quad$ and $\quad \frac{\not q+\not p_{i}+M_{i}}{\bar{D}_{p_{i}}}$,

respectively, with

$$
\begin{aligned}
\bar{D}_{p_{i}} & =\left(q+p_{i}\right)^{2}-M_{i}^{2}-\mu^{2}=\bar{q}^{2}-d_{i}, \\
\bar{q}^{2} & \equiv q^{2}-\mu^{2}, \quad d_{i} \equiv M_{i}^{2}-p_{i}^{2}-2\left(q \cdot p_{i}\right) .
\end{aligned}
$$

Secondly, UV infinities are isolated by a repeated use of the identity

$\frac{1}{\bar{D}_{p_{i}}}=\frac{1}{\bar{q}^{2}}+\frac{d_{i}}{\bar{q}^{2} \bar{D}_{p_{i}}}$

In fact-being $d_{i}$ is at most linear in $q$ - the second term in the r.h.s. of Eq. (3) is less UV divergent than the original denominator, so that UV divergences can be systematically moved to terms such as $1 / \bar{q}^{2}$, which depend only on $\mu$, and directly subtracted from the integrand. Schematically, dubbing $J$ the original integrand of an $\ell$-loop function, one has

$$
J\left(q_{1}, \ldots, q_{\ell}\right)=J_{\mathrm{INF}}\left(q_{1}, \ldots, q_{\ell}\right)+J_{\mathrm{F}, \ell}\left(q_{1}, \ldots, q_{\ell}\right),
$$

\footnotetext{
2 One-loop examples have been worked out in $[37,38]$.

${ }^{3} q$ denotes a generic integration momentum and $p_{i}$ and external momentum.
}

where $J_{\text {INF }}$ collects the UV divergent integrands. Then the FDR integral over $J$ is defined as ${ }^{4}$

$$
\begin{aligned}
& \int\left[d^{4} q_{1}\right] \ldots\left[d^{4} q_{\ell}\right] J\left(q_{1}, \ldots, q_{\ell}\right) \\
& \equiv \lim _{\mu \rightarrow 0} \int d^{4} q_{1} \ldots d^{4} q_{\ell} J_{\mathrm{F}, \ell}\left(q_{1}, \ldots q_{\ell}\right),
\end{aligned}
$$

where, due to the limit $\mu \rightarrow 0$, only a logarithmic dependence on $\mu$ remains, which can be traded for a dependence on the renormalization scale. ${ }^{5}$ Thus, FDR and normal integration coincide in a convergent integral, since no divergent part $J_{\mathrm{INF}}$ can be extracted from its integrand. Furthermore, the space-time is kept strictly four-dimensional also in divergent integrals—with $g^{\alpha \beta}=\operatorname{diag}(1,-1,-1,-1)$-because $\mu^{2}$ is nothing but the infinitesimal deformation needed to define the loop integrals ${ }^{6}$ and it is not generated by higherdimensional components of the integration momenta. This allows one to perform, in particular, the Dirac gamma algebra in four dimensions, with extra rules needed to keep gauge invariance, as explained in Sect. 2.4.

An explicit example of integrand FDR expansion ${ }^{7}$ at one loop is given by

$$
\begin{aligned}
\frac{q^{\alpha} q^{\beta}}{\bar{D}_{p_{0}} \bar{D}_{p_{1}}}= & {\left[\frac{q^{\alpha} q^{\beta}}{\bar{q}^{4}}\right]+\left(d_{0}+M_{1}^{2}-p_{1}^{2}\right)\left[\frac{q^{\alpha} q^{\beta}}{\bar{q}^{6}}\right]-2 p_{1 \gamma} } \\
& \times\left[\frac{q^{\alpha} q^{\beta} q^{\gamma}}{\bar{q}^{6}}\right]+4 p_{1 \gamma} p_{1 \delta}\left[\frac{q^{\alpha} q^{\beta} q^{\gamma} q^{\delta}}{\bar{q}^{8}}\right]+J_{\mathrm{F}, 1}^{\alpha \beta}(q),
\end{aligned}
$$

$$
\begin{aligned}
J_{\mathrm{F}, 1}^{\alpha \beta}(q)= & q^{\alpha} q^{\beta}\left(\frac{4\left(q \cdot p_{1}\right)^{2} d_{1}}{\bar{q}^{8} \bar{D}_{p_{1}}}\right. \\
& +\left(M_{1}^{2}-p_{1}^{2}\right) \frac{d_{0}+d_{1}-2\left(q \cdot p_{1}\right)}{\bar{q}^{6} \bar{D}_{p_{1}}} \\
& \left.-2 d_{0} \frac{\left(q \cdot p_{1}\right)}{\bar{q}^{6} \bar{D}_{p_{1}}}+\frac{d_{0}^{2}}{\bar{q}^{4} \bar{D}_{p_{0}} \bar{D}_{p_{1}}}\right),
\end{aligned}
$$

where $p_{0}=0$ and the terms in square brackets are proportional to UV divergent integrands. A two-loop example with

$$
\begin{aligned}
& \bar{D}_{1}=\bar{q}_{1}^{2}-m_{1}^{2}, \quad \bar{D}_{2}=\bar{q}_{2}^{2}-m_{2}^{2}, \quad \bar{D}_{12}=\bar{q}_{12}^{2}-m_{12}^{2}, \\
& q_{12}=q_{1}+q_{2}
\end{aligned}
$$

\footnotetext{
4 Throughout the paper FDR integration is denoted by the symbol $\left[d^{4} q_{i}\right]$.

5 see Sect. 2.3.

${ }^{6}$ Unlike in DR, the limit $\mu \rightarrow 0$ is taken outside integration [see Eq. (5)].

7 We denote the expansion of an integrand $J$ needed to bring it in the form of Eq. (4) as its FDR defining expansion.
} 
reads

$$
\begin{aligned}
\frac{1}{\bar{D}_{1} \bar{D}_{2} \bar{D}_{12}}= & {\left[\frac{1}{\bar{q}_{1}^{2} \bar{q}_{2}^{2} \bar{q}_{12}^{2}}\right]+m_{1}^{2}\left[\frac{1}{\bar{q}_{1}^{4} \bar{q}_{2}^{2} \bar{q}_{12}^{2}}\right] } \\
& +m_{2}^{2}\left[\frac{1}{\bar{q}_{1}^{2} \bar{q}_{2}^{4} \bar{q}_{12}^{2}}\right]+m_{12}^{2}\left[\frac{1}{\bar{q}_{1}^{2} \bar{q}_{2}^{2} \bar{q}_{12}^{4}}\right] \\
& +\frac{m_{1}^{4}}{\left(\bar{D}_{1} \bar{q}_{1}^{4}\right)}\left[\frac{1}{\bar{q}_{2}^{4}}\right]+\frac{m_{2}^{4}}{\left(\bar{D}_{2} \bar{q}_{2}^{4}\right)}\left[\frac{1}{\bar{q}_{1}^{4}}\right] \\
& +\frac{m_{12}^{4}}{\left(\bar{D}_{12} \bar{q}_{12}^{4}\right)}\left[\frac{1}{\bar{q}_{1}^{4}}\right]+J_{\mathrm{F}, 2}\left(q_{1}, q_{2}\right),
\end{aligned}
$$

where

$$
\begin{aligned}
J_{\mathrm{F}, 2}\left(q_{1}, q_{2}\right)= & -m_{1}^{4} \frac{q_{1}^{2}+2\left(q_{1} \cdot q_{2}\right)}{\left(\bar{D}_{1} \bar{q}_{1}^{4}\right) \bar{q}_{2}^{4} \bar{q}_{12}^{2}}-m_{2}^{4} \frac{q_{2}^{2}+2\left(q_{1} \cdot q_{2}\right)}{\bar{q}_{1}^{4}\left(\bar{D}_{2} \bar{q}_{2}^{4}\right) \bar{q}_{12}^{2}} \\
& -m_{12}^{4} \frac{q_{12}^{2}-2\left(q_{1} \cdot q_{12}\right)}{\bar{q}_{1}^{4} \bar{q}_{2}^{2}\left(\bar{D}_{12} \bar{q}_{12}^{4}\right)}+\frac{m_{1}^{2} m_{2}^{2}}{\left(\bar{D}_{1} \bar{q}_{1}^{2}\right)\left(\bar{D}_{2} \bar{q}_{2}^{2}\right) \bar{q}_{12}^{2}} \\
& +\frac{m_{1}^{2} m_{12}^{2}}{\left(\bar{D}_{1} \bar{q}_{1}^{2}\right) \bar{q}_{2}^{2}\left(\bar{D}_{12} \bar{q}_{12}^{2}\right)}+\frac{m_{2}^{2} m_{12}^{2}}{\bar{q}_{1}^{2}\left(\bar{D}_{2} \bar{q}_{2}^{2}\right)\left(\bar{D}_{12} \bar{q}_{12}^{2}\right)} \\
& +\frac{m_{1}^{2} m_{2}^{2} m_{12}^{2}}{\left(\bar{D}_{1} \bar{q}_{1}^{2}\right)\left(\bar{D}_{2} \bar{q}_{2}^{2}\right)\left(\bar{D}_{12} \bar{q}_{12}^{2}\right)} .
\end{aligned}
$$

Note that identities such as

$$
\begin{aligned}
\frac{1}{\bar{q}_{12}^{2}} & =\frac{1}{\bar{q}_{2}^{2}}-\frac{q_{1}^{2}+2\left(q_{1} \cdot q_{2}\right)}{\bar{q}_{2}^{2} \bar{q}_{12}^{2}}, \\
\frac{1}{\bar{q}_{2}^{2}} & =\frac{1}{\bar{q}_{1}^{2}}-\frac{q_{12}^{2}-2\left(q_{1} \cdot q_{12}\right)}{\bar{q}_{1}^{2} \bar{q}_{2}^{2}}
\end{aligned}
$$

are needed to extract the sub-divergences. Then the one- and two-loop FDR integrals over the integrands in Eqs. (6) and (8) read

$$
\begin{aligned}
& \int\left[d^{4} q\right] \frac{q^{\alpha} q^{\beta}}{\bar{D}_{p_{0}} \bar{D}_{p_{1}}}=\lim _{\mu \rightarrow 0} \int d^{4} q J_{\mathrm{F}, 1}^{\alpha \beta}(q), \\
& \int\left[d^{4} q_{1}\right]\left[d^{4} q_{2}\right] \frac{1}{\bar{D}_{1} \bar{D}_{2} \bar{D}_{12}}=\lim _{\mu \rightarrow 0} \int d^{4} q_{1} d^{4} q_{2} J_{\mathrm{F}, 2}\left(q_{1}, q_{2}\right) .
\end{aligned}
$$

It is important to realize that divergent tensor structures are fully subtracted from the original integrand, as in Eq. (6). ${ }^{8}$ Owing to the Lorentz invariance and four-dimensionality of this definition, irreducible tensors can be decomposed in terms of scalars. For example ${ }^{9}$

\footnotetext{
${ }^{8}$ It can be shown that FDR tensors are equivalent to DR tensors at one loop, but differences start at two loops and beyond [39].

9 The FDR defining expansion of $\frac{q_{1}^{\alpha} q_{1}^{\beta}}{\bar{D}_{1}^{3} \bar{D}_{2} \bar{D}_{12}}$ is given in Appendix C.
}

$$
\begin{aligned}
& \int\left[d^{4} q_{1}\right]\left[d^{4} q_{2}\right] \frac{q_{1}^{\alpha} q_{1}^{\beta}}{\bar{D}_{1}^{3} \bar{D}_{2} \bar{D}_{12}} \\
& =\lim _{\mu \rightarrow 0} \int d^{4} q_{1} d^{4} q_{2} q_{1}^{\alpha} q_{1}^{\beta} J_{D}\left(q_{1}, q_{2}\right), \\
& \quad \text { where } J_{D}\left(q_{1}, q_{2}\right)=\left\{\left(\frac{1}{\bar{q}_{1}^{6}}-\frac{1}{\bar{D}_{1}^{3}}\right) \frac{q_{1}^{2}+2\left(q_{1} \cdot q_{2}\right)}{\bar{q}_{2}^{4} \bar{q}_{12}^{2}}\right. \\
& \left.\quad+\frac{1}{\bar{D}_{1}^{3} \bar{q}_{2}^{2} \bar{D}_{12}}\left(\frac{m_{2}^{2}}{\bar{D}_{2}}+\frac{m_{12}^{2}}{\bar{q}_{12}^{2}}\right)\right\},
\end{aligned}
$$

can be rewritten as

$$
\begin{aligned}
& \int\left[d^{4} q_{1}\right]\left[d^{4} q_{2}\right] \frac{q_{1}^{\alpha} q_{1}^{\beta}}{\bar{D}_{1}^{3} \bar{D}_{2} \bar{D}_{12}} \\
& =\frac{g^{\alpha \beta}}{4} \int\left[d^{4} q_{1}\right]\left[d^{4} q_{2}\right] \frac{q_{1}^{2}}{\bar{D}_{1}^{3} \bar{D}_{2} \bar{D}_{12}},
\end{aligned}
$$

with

$$
\begin{aligned}
& \int\left[d^{4} q_{1}\right]\left[d^{4} q_{2}\right] \frac{q_{1}^{2}}{\bar{D}_{1}^{3} \bar{D}_{2} \bar{D}_{12}} \\
& =\lim _{\mu \rightarrow 0} \int d^{4} q_{1} d^{4} q_{2} q_{1}^{2} J_{D}\left(q_{1}, q_{2}\right) .
\end{aligned}
$$

Finally, polynomials in the integration variables represent a limiting case of Eq. (4), in which

$J_{\mathrm{F}, \ell}\left(q_{1}, \ldots, q_{\ell}\right)=0$.

As a consequence

$$
\int\left[d^{4} q\right]\left(\bar{q}^{2}\right)^{\alpha}=0
$$

for any integer $\alpha \geq 0$.

\subsection{Shift invariance and uniqueness}

FDR integrals are invariant under the shift of any integration variable. This can easily be proven by using the fact that they can be thought of as finite differences of shift-invariant dimensionally regulated ${ }^{10}$ divergent integrals [see Eq. (4)]

$$
\begin{aligned}
\int\left[d^{4} q_{1}\right] \ldots\left[d^{4} q_{\ell}\right] J\left(q_{1}, \ldots, q_{\ell}\right) \\
=\lim _{\mu \rightarrow 0} \mu_{\mathrm{R}}^{-\ell \epsilon}\left(\int d^{n} q_{1} \ldots d^{n} q_{\ell} J\left(q_{1}, \ldots, q_{\ell}\right)\right. \\
\left.\quad-\int d^{n} q_{1} \ldots d^{n} q_{\ell} J_{\mathrm{INF}}\left(q_{1}, \ldots, q_{\ell}\right)\right) .
\end{aligned}
$$

The explicit demonstration is given in Appendix A. A corollary to this theorem is the uniqueness of the definition in Eq. (5). In fact, the subtracted integrands in $J_{\mathrm{INF}}\left(q_{1}, \ldots, q_{\ell}\right)$

\footnotetext{
${ }^{10}$ Here and in the following $n=4+\epsilon$ and $\mu_{\mathrm{R}}$ is the renormalization scale.
} 
are unambiguously determined by the UV content of the original integrand, the only possible ambiguity being shifts of the loop momenta in $J\left(q_{1}, \ldots, q_{\ell}\right)$, which, however, produce the same FDR integral.

Equation (17) also demonstrates that whenever DR loop integrals are known, their FDR counterparts can also be computed.

\subsection{Independence of the cutoff}

As a result of the subtraction of the divergent integrands, non integrable powers of $1 / \bar{q}^{2}$ are developed in $J_{\mathrm{F}, \ell}\left(q_{1}, \ldots, q_{\ell}\right)$. Such IR poles get regulated by the $\mu^{2}$ propagator prescription, which gives a meaning to the r.h.s. of Eq. (5). Thus, the original UV cutoff is traded for an IR one: $\mu$. Here we show that FDR integrals depend at most logarithmically on $\mu$. Furthermore, $\mu$ can be traded for the renormalization scale $\mu_{\mathrm{R}}$, rendering the definition of the FDR integration independent of any cutoff.

We start from Eq. (17). Since the first term in its r.h.s. is the original DR regulated integral it does not depend on $\mu$, in the limit $\mu \rightarrow 0 .{ }^{11}$ On the other hand, polynomially divergent integrands in $J_{\mathrm{INF}}$ cannot contribute either, because they generate polynomials in $\mu$. Therefore, the $\mu$ dependence in the 1.h.s. is entirely due to powers of $\ln \left(\mu / \mu_{\mathrm{R}}\right)$ created by the subtraction of the logarithmically divergent integrals. If one redefines FDR integrals without subtracting such logarithms, no dependence on $\mu$ is produced. This is equivalent to the operation of adding back all $\ln \left(\mu / \mu_{\mathrm{R}}\right) \mathrm{s}$ to the 1.h.s. of Eq. (17). Then the limit $\mu \rightarrow 0$ can be taken, $\mu$ becomes $\mu_{\mathrm{R}}$, and no cutoff is left. The identification $\mu=\mu_{\mathrm{R}}$ after $\lim _{\mu \rightarrow 0}$ is understood in all FDR integrals appearing in this paper.

\subsection{Keeping gauge invariance}

Now we discuss how gauge invariance is preserved in FDR. Our starting point is the existence of graphical proofs of the Ward-Slavnov-Taylor identities [40], in which the correct relations among Green's functions are demonstrated-at any loop order-directly at the level of Feynman diagrams. Such proofs are valid under two circumstances:

- divergent loop integrals should be defined in a way that shifting the integration momenta is possible as if they were convergent ones [41];

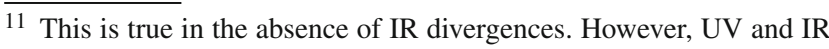
infinities simultaneously occur only in scale-less integrals, which vanish in FDR [see Sect. 2.7].
}

- cancelations between numerators and denominators should be preserved. ${ }^{12}$

Since the first property has been already proven, we concentrate here on the second requirement, which we study by means of a two-loop example.

Consider the scalar integral

$\int\left[d^{4} q_{1}\right]\left[d^{4} q_{2}\right] \frac{1}{\bar{D}_{1}^{2} \bar{D}_{2} \bar{D}_{12}}$.

To define it in FDR, it is necessary to make explicit the $\mu^{2}$ dependence in its denominators, ${ }^{13}$ which amounts to the replacement

$q_{i}^{2} \rightarrow q_{i}^{2}-\mu^{2}$

However, this change should be performed without altering the cancelations which ensure that the same result is obtained both by simplifying the reducible numerators before computing the integrals and by working out the integrals without simplifying the numerators.

That happens only if

1. any $q_{i}^{2}$ generated by Feynman rules in the numerator of a diagram $^{14}$ is also changed as in Eq. (19);

2. simplifications at the integrand level are possible, such as

$$
\begin{aligned}
& \int {\left[d^{4} q_{1}\right]\left[d^{4} q_{2}\right] \frac{q_{1}^{2}-m_{1}^{2}-\mu^{2}}{\bar{D}_{1}^{3} \bar{D}_{2} \bar{D}_{12}} } \\
&=\int\left[d^{4} q_{1}\right]\left[d^{4} q_{2}\right] \frac{1}{\bar{D}_{1}^{2} \bar{D}_{2} \bar{D}_{12}} .
\end{aligned}
$$

Either way, integrals with $\mu$ in the numerator appearwhich we dub extra integrals - that need to be properly defined. For instance, since an explicit computation gives

$$
\int\left[d^{4} q_{1}\right]\left[d^{4} q_{2}\right] \frac{q_{1}^{2}-m_{1}^{2}}{\bar{D}_{1}^{3} \bar{D}_{2} \bar{D}_{12}} \neq \int\left[d^{4} q_{1}\right]\left[d^{4} q_{2}\right] \frac{1}{\bar{D}_{1}^{2} \bar{D}_{2} \bar{D}_{12}}
$$

one deduces that ${ }^{15}$

\footnotetext{
12 Quoting Martinus Veltman [42]: Gauge invariance implies a tight interplay between the numerator of an integrand and its denominator. Changing either of the two will generally destroy gage invariance...

13 See Eq. (7).

14 Such $q^{2}$ terms are created, for instance, when $\left(q+p_{i}\right)^{\alpha}\left(q+p_{i}\right)^{\beta} / M_{i}^{2}$ and $\not q+\not p_{1}+M_{i}$ in Eq. (1) are multiplied by $g_{\alpha \beta}$ and $\phi$, respectively, before tensor reduction.

15 The r.h.s. of Eq. (22) vanishes because FDR integrals are at most logarithmic in $\mu$.
} 


$$
\begin{aligned}
& \int\left[d^{4} q_{1}\right]\left[d^{4} q_{2}\right] \frac{\mu^{2}}{\bar{D}_{1}^{3} \bar{D}_{2} \bar{D}_{12}} \\
& \quad \neq \lim _{\mu \rightarrow 0} \mu^{2} \int\left[d^{4} q_{1}\right]\left[d^{4} q_{2}\right] \frac{1}{\bar{D}_{1}^{3} \bar{D}_{2} \bar{D}_{12}}=0 .
\end{aligned}
$$

In fact, a non-zero contribution must be added to the 1.h.s. of Eq. (21) to produce the r.h.s. of Eq. (20). The right cancelation occurs if the denominators $1 / \bar{D}_{1}^{3} \bar{D}_{2} \bar{D}_{12}$ are expanded in front of $\mu^{2}$ as if it was a $q_{1}^{2}$, namely as in Eq. (12) ${ }^{16}$ :

$$
\begin{aligned}
& \int\left[d^{4} q_{1}\right]\left[d^{4} q_{2}\right] \frac{\left.\mu^{2}\right|_{1}}{\bar{D}_{1}^{3} \bar{D}_{2} \bar{D}_{12}} \\
& =\lim _{\mu \rightarrow 0} \int d^{4} q_{1} d^{4} q_{2} \mu^{2} J_{D}\left(q_{1}, q_{2}\right) .
\end{aligned}
$$

By using this definition, Eq. (20) directly follows from the FDR defining expansion of its two sides. Note that the index 1 in $\left.\mu^{2}\right|_{1}$ only denotes the expansion to be used: although only one kind of $\mu^{2}$ exists

$$
\begin{aligned}
& \int\left[d^{4} q_{1}\right]\left[d^{4} q_{2}\right] \frac{\left.\mu^{2}\right|_{1}}{\bar{D}_{1}^{3} \bar{D}_{2} \bar{D}_{12}}, \int\left[d^{4} q_{1}\right]\left[d^{4} q_{2}\right] \frac{\left.\mu^{2}\right|_{2}}{\bar{D}_{1}^{3} \bar{D}_{2} \bar{D}_{12}} \\
& \quad \text { and } \int\left[d^{4} q_{1}\right]\left[d^{4} q_{2}\right] \frac{\left.\mu^{2}\right|_{12}}{\bar{D}_{1}^{3} \bar{D}_{2} \bar{D}_{12}}
\end{aligned}
$$

are in general different, because they are defined by expanding

$$
\begin{aligned}
& \int\left[d^{4} q_{1}\right]\left[d^{4} q_{2}\right] \frac{q_{1}^{2}}{\bar{D}_{1}^{3} \bar{D}_{2} \bar{D}_{12}}, \int\left[d^{4} q_{1}\right]\left[d^{4} q_{2}\right] \frac{q_{2}^{2}}{\bar{D}_{1}^{3} \bar{D}_{2} \bar{D}_{12}} \\
& \quad \text { and } \int\left[d^{4} q_{1}\right]\left[d^{4} q_{2}\right] \frac{q_{12}^{2}}{\bar{D}_{1}^{3} \bar{D}_{2} \bar{D}_{12}},
\end{aligned}
$$

respectively.

The described procedure is completely general: the extra integrals are defined by the FDR expansion of the integrals obtained by replacing $\left.\mu\right|_{i} \rightarrow q_{i}$. As a consequence, the $\left.\mu\right|_{i}$ in the numerator are sensitive to changes of variables. For example, if $q_{1} \rightarrow q_{1}-q_{2}$ and $q_{2} \rightarrow-q_{2}$,

$$
\begin{aligned}
\int\left[d^{4} q_{1}\right]\left[d^{4} q_{2}\right] \frac{\left.\mu^{2}\right|_{12}}{\bar{D}_{1}^{3} \bar{D}_{2} \bar{D}_{12}} \\
\quad \rightarrow \int\left[d^{4} q_{1}\right]\left[d^{4} q_{2}\right] \frac{\left.\mu^{2}\right|_{1}}{\bar{D}_{1} \bar{D}_{2} \bar{D}_{12}^{3}} .
\end{aligned}
$$

\footnotetext{
${ }^{16}$ It is interesting to study how a finite contribution is generated by the definition in Eq. (23). In $J_{D}\left(q_{1}, q_{2}\right)$$$
\int d^{4} q_{1} d^{4} q_{2} \frac{q_{1}^{2}+2\left(q_{1} \cdot q_{2}\right)}{\bar{q}_{1}^{6} \bar{q}_{2}^{4} \bar{q}_{12}^{2}} \sim \frac{1}{\mu^{2}}
$$

thus

$\int\left[d^{4} q_{1}\right]\left[d^{4} q_{2}\right] \frac{\left.\mu^{2}\right|_{1}}{\bar{D}_{1}^{3} \bar{D}_{2} \bar{D}_{12}}=\lim _{\mu \rightarrow 0} \mu^{2} \int d^{4} q_{1} d^{4} q_{2} \frac{q_{1}^{2}+2\left(q_{1} \cdot q_{2}\right)}{\bar{q}_{1}^{6} \bar{q}_{2}^{4} \bar{q}_{12}^{2}}$

produces a finite constant when $\mu \rightarrow 0$. The value of this integral is given in Sect. 3.1.
}

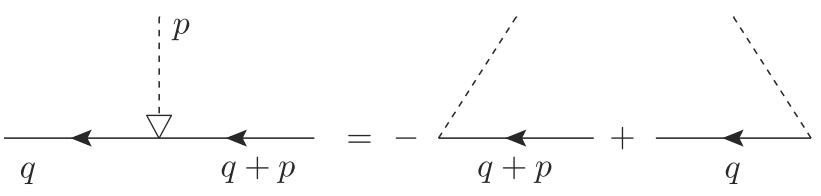

Fig. 1 Graphical representation of the Feynman identity in Eq. (29). The dashed line represents a scalar photon

Extra integrals can be computed either directly, by considering the finite part $J_{D}\left(q_{1}, q_{2}\right)$ of the relevant denominator expansion - as done in Eq. (23)_or indirectly, by rewriting $J_{D}\left(q_{1}, q_{2}\right)$ as a difference between the original integrand and its subtracted pieces. This second way is usually more convenient, because the original integral does not contribute in the limit $\mu \rightarrow 0$. For example, Eq. (125) gives

$$
\begin{aligned}
& \int\left[d^{4} q_{1}\right]\left[d^{4} q_{2}\right] \frac{\left.\mu^{2}\right|_{1}}{\bar{D}_{1}^{3} \bar{D}_{2} \bar{D}_{12}} \\
& =-\lim _{\mu \rightarrow 0} \mu^{2} \int d^{4} q_{1} d^{4} q_{2}\left(\frac{1}{\bar{q}_{1}^{6} \bar{q}_{2}^{2} \bar{q}_{12}^{2}}-\frac{1}{\bar{q}_{1}^{6}} \frac{1}{\bar{q}_{2}^{4}}\right),
\end{aligned}
$$

which coincides with the result in footnote 16 .

Finally, extra integrals give the possibility to rewrite tensors in terms of scalars plus constants. For instance, Eq. (13) produces

$$
\begin{aligned}
\int & {\left[d^{4} q_{1}\right]\left[d^{4} q_{2}\right] \frac{q_{1}^{\alpha} q_{1}^{\beta}}{\bar{D}_{1}^{3} \bar{D}_{2} \bar{D}_{12}} } \\
= & \frac{g^{\alpha \beta}}{4}\left\{\int\left[d^{4} q_{1}\right]\left[d^{4} q_{2}\right] \frac{1}{\bar{D}_{1}^{2} \bar{D}_{2} \bar{D}_{12}}\right. \\
& +m_{1}^{2} \int\left[d^{4} q_{1}\right]\left[d^{4} q_{2}\right] \frac{1}{\bar{D}_{1}^{3} \bar{D}_{2} \bar{D}_{12}} \\
& \left.+\int\left[d^{4} q_{1}\right]\left[d^{4} q_{2}\right] \frac{\left.\mu^{2}\right|_{1}}{\bar{D}_{1}^{3} \bar{D}_{2} \bar{D}_{12}}\right\}
\end{aligned}
$$

Decompositions like this will be extensively used in the calculation presented in Sect. 3.

Having studied the general mechanism of the gauge cancelations in FDR, we further elucidate it by means of the process investigated in this paper, namely $H \rightarrow \gamma \gamma$ mediated by a fermion with mass $m$. In this case the proof of gauge invariance relies on the graphical equivalence depicted in Fig. 1, which, in turn, is realized by the Feynman identity

$\frac{\not 1+m}{D} \not p \frac{q 1+\not p+m}{D_{p}}=\frac{\not 1+m}{D}-\frac{\not 1+\not p+m}{D_{p}}$,

where

$D=q^{2}-m^{2}$ and $D_{p}=(q+p)^{2}-m^{2}$.

Consider now the generic $\ell$-loop amplitude in Fig. 2. 


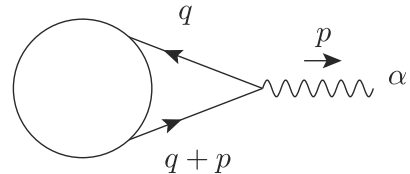

Fig. 2 Generic $\ell$-loop amplitude with an external photon with momentum $p$. The blob stands for the rest of the amplitude and $q$ is an integration momentum.

Its integrand reads

$$
\begin{aligned}
& \epsilon_{\alpha}(p) J^{\alpha}\left(q, \ldots, q_{\ell}\right)=\epsilon_{\alpha}(p) \sum_{i} \frac{1}{D D_{p}} \\
& \quad \times \operatorname{Tr}\left[(\not q+m) \gamma^{\alpha}(\not q+\not p+m)\left(\Gamma_{o}^{i}+\Gamma_{e}^{i}\right)\right],
\end{aligned}
$$

where the sum is over all contributing Feynman diagrams, and $\Gamma_{o}^{i}\left(\Gamma_{e}^{i}\right)$ is proportional to a product of an odd (even) number of gamma matrices. Gauge invariance requires that

$p_{\alpha} \int\left[d^{4} q\right] \cdots\left[d^{4} q_{\ell}\right] \bar{J}^{\alpha}\left(q, \ldots, q_{\ell}\right)=0$,

where $\bar{J}^{\alpha}$ is the integrand in Eq. (31) regulated $a$ la FDR by replacing $q_{i}^{2} \rightarrow q_{i}^{2}-\mu^{2}$ in both numerators and denominators. Equation (32) can be directly proven at the integrand level. With this aim, we first concentrate on the replacements responsible for the conservation of the specific current in Fig. 2:

$$
\begin{aligned}
J^{\alpha} \rightarrow J^{\prime \alpha}= & \sum_{i} \frac{1}{\bar{D} \bar{D}_{p}}\left(\operatorname{Tr}\left[\bar{d} \gamma^{\alpha} \not{\phi} \Gamma_{o}^{i}\right]+\operatorname{Tr}\left[\not \alpha \gamma^{\alpha} \not p \Gamma_{o}^{i}\right]\right. \\
& +m^{2} \operatorname{Tr}\left[\gamma^{\alpha} \Gamma_{o}^{i}\right]+m \operatorname{Tr}\left[\gamma^{\alpha}(\phi+\not p) \Gamma_{e}^{i}\right] \\
& \left.+m \operatorname{Tr}\left[\not d \gamma^{\alpha} \Gamma_{e}^{i}\right]\right),
\end{aligned}
$$

where the loop denominators in $\Gamma_{o, e}^{i}$ are also barred. In the previous equation

$\Phi=\not d \pm \mu$

has the effect of changing $q^{2}$ to $\bar{q}^{2}$ in the first trace. Thus, when contracting with $p$, it is possible to reconstruct and cancel denominators

$$
\begin{aligned}
p_{\alpha} J^{\prime \alpha}= & \sum_{i} \frac{1}{\bar{D}}\left(\operatorname{Tr}\left[\not \Gamma_{o}^{i}\right]+m \operatorname{Tr}\left[\Gamma_{e}^{i}\right]\right) \\
& -\frac{1}{\bar{D}_{p}}\left(\operatorname{Tr}\left[(\not q+\not p) \Gamma_{o}^{i}\right]+m \operatorname{Tr}\left[\Gamma_{e}^{i}\right]\right),
\end{aligned}
$$

in agreement with the Feynman identity in Eq. (29). After that

$p_{\alpha} \int\left[d^{4} q\right] \cdots\left[d^{4} q_{\ell}\right] J^{\prime \alpha}\left(q, \ldots, q_{\ell}\right)=0$

directly follows from the shift invariance properties of the loop integrals, as in DR. We explicitly tested Eq. (36) up to two loops in $H \rightarrow \gamma \gamma$.
With more photons, replacements as in Eq. (34) have to be performed for all integration momenta appearing in the trace. ${ }^{17}$ The one-loop prescription is that defined in [38]: given a fermionic string, one chooses arbitrarily the sign of $\mu$ within the first $\bar{q}$; the sign of the subsequent one is opposite, if an even number of $\gamma$-matrices occur between the two $\bar{q} s$, and it is the same otherwise. ${ }^{18}$ This rule is sufficient in the presence of one fermion line only, as in the calculation at hand. With two or more lines, and no summation indices among them, each fermion string can be separately treated as described. If sums occur, after applying the above algorithm, extra $\mu^{2}$ terms need to be extracted according to the following procedure:

$$
\begin{aligned}
& \operatorname{Tr}\left[\ldots \not \Gamma^{(n)} \gamma_{\alpha}\right] \operatorname{Tr}\left[\ldots \not \phi \Gamma^{(m)} \gamma^{\alpha}\right] \\
& \quad \rightarrow \operatorname{Tr}\left[\ldots \not \phi \Gamma^{(n)} \gamma_{\alpha}\right] \operatorname{Tr}\left[\ldots \not \Gamma^{(m)} \gamma^{\alpha}\right] \\
& \quad-(-1)^{(n+m)} \mu^{2} \operatorname{Tr}\left[\ldots \Gamma^{(n)}\right] \operatorname{Tr}\left[\ldots \Gamma^{(m)}\right],
\end{aligned}
$$

where $\Gamma^{(k)}$ represents a string of $k$ gamma matrices. Equation (37) is proven by noting that $n(\mathrm{~m})$ anticommutations are needed to bring $\phi$ close to $\gamma_{\alpha}\left(\gamma^{\alpha}\right)$ and can easily be checked by taking the traces and substituting $q^{2} \rightarrow q^{2}-\mu^{2}$. As an example of such rules, the integrand of the one-loop $H \rightarrow \gamma\left(p_{1}\right) \gamma\left(-p_{2}\right)$ amplitude is proportional to

$$
\begin{aligned}
J^{\alpha \beta}(q)= & \frac{1}{D D_{p_{1}} D_{p_{2}}} \operatorname{Tr}\left[(\not q+m) \gamma^{\alpha}\left(\not q+\not p_{1}+m\right)\right. \\
& \left.\times\left(\not 1+\not p_{2}+m\right) \gamma^{\beta}\right],
\end{aligned}
$$

and its FDR regulated version reads

$$
\begin{aligned}
\bar{J}^{\alpha \beta}(q)= & \frac{1}{\bar{D} \bar{D}_{p_{1}} \bar{D}_{p_{2}}}\left(\operatorname { T r } \left[(\not q+m) \gamma^{\alpha}\left(\not q+\not p_{1}+m\right)\right.\right. \\
& \left.\left.\times\left(\not q+\not p_{2}+m\right) \gamma^{\beta}\right]+m \mu^{2} \operatorname{Tr}\left[\gamma^{\alpha} \gamma^{\beta}\right]\right),
\end{aligned}
$$

which satisfies the Ward identities

$p_{1 \alpha} \int\left[d^{4} q\right] \bar{J}^{\alpha \beta}(q)=p_{2 \beta} \int\left[d^{4} q\right] \bar{J}^{\alpha \beta}(q)=0$.

We emphasize that there is nothing mysterious in Eq. (39): the same result is obtained by computing the trace in Eq. (38) and replacing $q^{2} \rightarrow \bar{q}^{2}$. The advantage of Eq. (39) is that it permits a trivial proof of the Ward identities at the integrand level.

\footnotetext{
17 Sums over internal indices first have to be worked out.

18 If chirality matrices are involved, a gauge invariant treatment requires their anticommutation at the beginning (or the end) of open strings before replacing $\phi \rightarrow \Phi_{1}$. In the case of closed loops, $\gamma_{5}$ should be put next to the vertex corresponding to a potential non-conserved current. This reproduces the correct coefficient of the triangular anomaly [30].
} 
The corresponding procedure at two loops is better explained with an example. Consider the trace

$T^{\alpha \beta}=\operatorname{Tr}\left[\phi_{1} \gamma^{\alpha} \phi_{1} \phi_{12} \gamma^{\beta} \phi_{22}\right]$,

which contributes to the second diagram of Fig. 5. Its FDR counterpart reads

$$
\begin{aligned}
\bar{T}^{\alpha \beta}= & T^{\alpha \beta}+\left.\mu^{2}\right|_{1} \operatorname{Tr}\left[\gamma^{\alpha} \phi_{2} \gamma^{\beta} \phi_{22}\right]+\left.\mu^{2}\right|_{2} \operatorname{Tr}\left[\gamma^{\alpha} \phi_{1} \gamma^{\beta} \phi_{1}\right] \\
& +\left.\left.\mu^{2}\right|_{1} \mu^{2}\right|_{2} \operatorname{Tr}\left[\gamma^{\alpha} \gamma^{\beta}\right]-16 \tilde{\mu}_{12}^{2} q_{1}^{\alpha} q_{2}^{\beta},
\end{aligned}
$$

with

$\tilde{\mu}_{12}^{2}=\frac{1}{2}\left(\left.\mu^{2}\right|_{12}-\left.\mu^{2}\right|_{1}-\left.\mu^{2}\right|_{2}\right)$.

Equation (42) is obtained from Eq. (41) by using-one after the other-the one-loop replacements $\phi_{1} \rightarrow \Phi_{1}$ and $\phi_{2} \rightarrow \Phi_{2}$, which generate the terms proportional to $\left.\mu^{2}\right|_{1}$ and $\left.\mu^{2}\right|_{2}$. The $\tilde{\mu}_{12}^{2}$ contribution originates, instead, from the substitution

$\left(q_{1} \cdot q_{2}\right)=\frac{1}{2}\left(q_{12}^{2}-q_{1}^{2}-q_{2}^{2}\right) \rightarrow \frac{1}{2}\left(\bar{q}_{12}^{2}-\bar{q}_{1}^{2}-\bar{q}_{2}^{2}\right)$,

and is obtained by simultaneously barring $\phi_{1}$ and $\phi_{2}$ in Eq. (41) (with the same rule used at one loop to determine the sign of $\left.\mu\right|_{i}$ inside each $\phi_{i}$, without distinguishing between $\phi_{1}$ and $\left.\phi_{2}\right)$ and subtracting the $\left.\mu^{2}\right|_{i}$ terms already calculated. What is left is, by construction, proportional to powers of $\left.\left.\mu\right|_{1} \mu\right|_{2} \equiv \tilde{\mu}_{12}^{2}$ and gives the last term in Eq. (42). Once
In practical cases it is often convenient to simplify reducible numerators before computing the loop integrals. In that way, only irreducible tensors appear and extra integrals are just produced by tensor decomposition, as in Eq. (28). This is the strategy of the calculation presented in Sect. 3.

\subsection{FDR versus DR}

The proof that DR preserves gauge invariance and unitarity relies on the possibility of introducing order-by-order local counterterms in the Lagrangian $\mathcal{L}$. On the contrary, FDR makes no reference to $\mathcal{L}$. In this subsection we use the simple two-loop QED example of [43] to comment on the conceptual differences between the two approaches.

Consider a DR calculation of the one-loop photon selfenergy

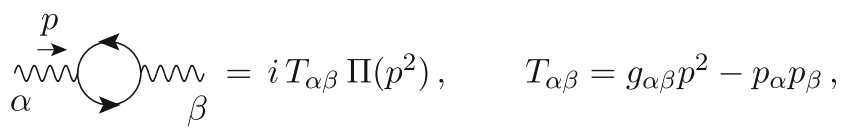

with

$$
\begin{aligned}
\Pi\left(p^{2}\right) & =\frac{1}{\epsilon} \Pi_{-1}+\Pi_{0}+\epsilon \Pi_{1}, \\
\Pi_{0} & =\frac{e^{2}}{2 \pi^{2}} \int_{0}^{1} d x x(1-x) \ln \frac{m^{2}-p^{2} x(1-x)}{\mu_{\mathrm{R}}^{2}} .
\end{aligned}
$$

Then, at two loops and up to terms $\mathcal{O}(\epsilon)$,

$$
m u=i T_{\alpha \beta} \Pi^{2}\left(p^{2}\right)=i T_{\alpha \beta}\left(\frac{\Pi_{-1}^{2}}{\epsilon^{2}}+\frac{2 \Pi_{-1} \Pi_{0}}{\epsilon}+\Pi_{0}^{2}+2 \Pi_{-1} \Pi_{1}\right) .
$$

again, $\bar{T}^{\alpha \beta}$ is equivalent to the replacements

$q_{1}^{2} \rightarrow \bar{q}_{1}^{2}, \quad q_{2}^{2} \rightarrow \bar{q}_{2}^{2}, \quad\left(q_{1} \cdot q_{2}\right) \rightarrow \frac{1}{2}\left(\bar{q}_{12}^{2}-\bar{q}_{1}^{2}-\bar{q}_{2}^{2}\right)$,

in the original trace $T^{\alpha \beta}$. Thus, the $\left.\mu^{2}\right|_{i}$ ensure the right cancelations leading to the fulfillment of the Ward identities. We explicitly checked that the two-loop $H \rightarrow \gamma\left(p_{1}\right) \gamma\left(-p_{2}\right)$ integrand $\bar{J}^{\alpha \beta}\left(q_{1}, q_{2}\right)$, constructed as described, satisfies
Simply removing the poles from the last expression gives $\Pi_{0}^{2}+2 \Pi_{-1} \Pi_{1}$, which is not the right result because it violates unitarity. As is well known, the correct procedure to undertake in DR is to renormalize order by order, i.e. to add one-loop counterterms in $\mathcal{L}$ such that

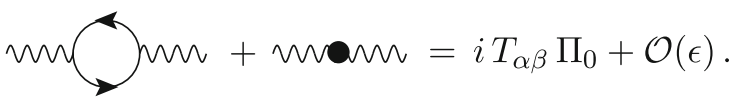

Thus

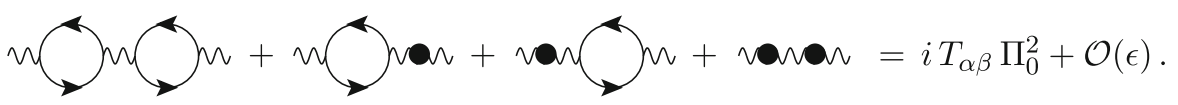

$$
\begin{aligned}
& p_{1 \alpha} \int\left[d^{4} q_{1}\right] \int\left[d^{4} q_{2}\right] \bar{J}^{\alpha \beta}\left(q_{1}, q_{2}\right) \\
& =p_{2 \beta} \int\left[d^{4} q_{1}\right] \int\left[d^{4} q_{2}\right] \bar{J}^{\alpha \beta}\left(q_{1}, q_{2}\right)=0 .
\end{aligned}
$$

In FDR, the divergences are subtracted at the level of the definition of the loop integration, so that the product of two one-loop diagrams is simply the product of the two finite parts, with no need of introducing extra interactions in $\mathcal{L}$. Thus, one directly obtains 


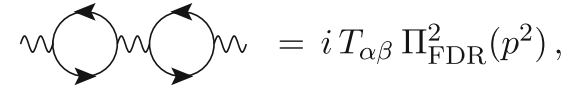

with $\Pi_{\mathrm{FDR}}\left(p^{2}\right)=\Pi_{0}$. This difference can also be understood from the DR $\leftrightarrow$ FDR naive correspondence

$$
\begin{aligned}
\epsilon & \leftrightarrow \mu \\
\frac{1}{\epsilon} & \leftrightarrow \ln \mu,
\end{aligned}
$$

which gives $\lim _{\epsilon \rightarrow 0} \epsilon / \epsilon=1$, while $\lim _{\mu \rightarrow 0} \mu \ln \mu=0$.

From all that it is manifest that spurious $\epsilon / \epsilon$ terms such as $\Pi_{-1} \Pi_{1}-$ which need to be kept under control in DR by the order-by-order renormalization-never appear in FDR. The result of an FDR calculation typically depends on the parameters contained in $\mathcal{L}$, and a (finite) global renormalization is needed only to link them to experimental measurements at the desired perturbative accuracy. In particular —and in contrast with DR-no renormalization is necessary when no parameter appears in the final result. This is the case of the calculation presented in Sect. 3 .

\subsection{FDR versus FDH}

In this subsection we discuss the differences between FDR and the Four-Dimensional Helicity scheme (FDH) of [44], which is equivalent to dimensional reduction [45] at one loop. FDH is a variant of DR, in which gauge cancelations are kept by integrating all momentum integrals over $n$-component momenta and considering any $g^{\alpha \beta}$ resulting from the integration as $n$-dimensional. Observed external states are treated in four dimensions (preserving supersymmetry) and unobserved internal ones are defined in such a way that the contraction $q^{\alpha} q^{\beta} g_{\alpha \beta}=q^{2}$ gives rise to an $n$-dimensional object when $q$ is an integration momentum. If $q^{2}$ is split into fourdimensional $\left(q_{4}^{2}\right)$ and $\epsilon$-dimensional $\left(\tilde{q}^{2}\right)$ components,

$q^{2}=q_{4}^{2}+\tilde{q}^{2}$,

and $\tilde{q}^{2}$ is identified with $-\mu^{2}$, there is a formal equivalenceat the integrand level-between the procedures used by FDR and FDH to determine the $\mu^{2}$ pieces $[46,47]$. However, differences start when integrating. FDR integration is defined in a way that non-local sub-divergences are subtracted right away, as in the second line of Eq. (8), while in FDH sub-divergences are compensated by counterterms added at a previous renormalization stage, as in any conventional subtraction scheme. It is exactly this peculiarity that makes it possible to avoid an order-by-order renormalization in FDR.

As a consequence of this dissimilarity, integrals containing $\mu^{2}$ give different results, at two loops and beyond, when computed in FDR and FDH. For example
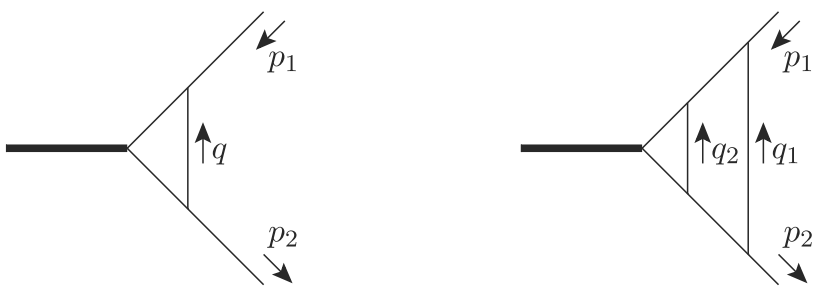

Fig. 3 Examples of massless one-loop and two-loop scalar integrals. Thin lines represent massless scalar propagators and $p_{1}^{2}=p_{2}^{2}=0$

$$
\begin{aligned}
& \int\left[d^{4} q_{1}\right]\left[d^{4} q_{2}\right] \frac{\left.\mu^{2}\right|_{1}}{\left(\bar{q}_{1}^{2}-m^{2}\right)^{2}\left(\bar{q}_{1}^{2}-m^{2}\right)^{2}\left(\bar{q}_{12}^{2}-m^{2}\right)} \\
& =\pi^{4}\left(\frac{2}{3} f+\frac{1}{2} \ln \frac{m^{2}}{\mu_{\mathrm{R}}^{2}}\right),
\end{aligned}
$$

with $f$ defined in Eq. (130), while

$$
\begin{aligned}
& \int d^{n} q_{1} d^{n} q_{2} \frac{-\tilde{q}_{1}^{2}}{\left(q_{1}^{2}-m^{2}\right)^{2}\left(q_{1}^{2}-m^{2}\right)^{2}\left(q_{12}^{2}-m^{2}\right)} \\
& =\pi^{4}\left(\frac{1}{2 \epsilon}-\frac{3}{8}+\frac{1}{2} \ln \frac{m^{2}}{\mu_{\mathrm{R}}^{2}}\right. \\
& \left.+\frac{\gamma_{E}+\ln \pi}{2}\right)+\mathcal{O}(\epsilon) .
\end{aligned}
$$

Only at one loop, because no sub-divergences are present, FDR and FDH coincide, as observed in [37]. For instance,

$$
\int\left[d^{4} q\right] \frac{\mu^{2}}{\left(q^{2}-M^{2}\right)^{3}}=\int d^{n} q \frac{-\tilde{q}^{2}}{\left(q^{2}-M^{2}\right)^{3}}=\frac{i \pi^{2}}{2} .
$$

\subsection{Infrared divergences}

Although the process $H \rightarrow \gamma \gamma$ is free of IR infinities, we devote this subsection to an illustration of how soft and collinear singularities can be treated compatibly with FDR. We first discuss divergences in the virtual contribution, and then show how they are matched by a particular treatment of the real radiation.

As for the loop integration, the definition in Eq. (5) can be maintained also in the presence of IR singularities. For instance, the FDR versions of the massless one- and twoloop scalar integrals in Fig. 3 read

$$
\begin{aligned}
& \int\left[d^{4} q\right] J^{(1)}\left(q, \mu^{2}\right)=\lim _{\mu \rightarrow 0} \int d^{4} q J^{(1)}\left(q_{1}, \mu^{2}\right) \text { and } \\
& \int\left[d^{4} q_{1}\right]\left[d^{4} q_{2}\right] J^{(2)}\left(q_{1}, q_{2}, \mu^{2}\right) \\
& =\lim _{\mu \rightarrow 0} \int d^{4} q_{1} d^{4} q_{2} J^{(2)}\left(q_{1}, q_{2}, \mu^{2}\right),
\end{aligned}
$$

respectively, with ${ }^{19}$

\footnotetext{
$\overline{19 J^{(1)} \text { and } J^{(2)}}$ are not UV subtracted since they produce UV convergent integrals.
} 


$$
\begin{aligned}
& J^{(1)}\left(q, \mu^{2}\right)=\frac{1}{\bar{D}_{0}(q) \bar{D}_{p_{1}}(q) \bar{D}_{p_{2}}(q)}, \\
& J^{(2)}\left(q_{1}, q_{2}, \mu^{2}\right)=J^{(1)}\left(q_{1}, \mu^{2}\right) \frac{1}{\bar{D}_{0}\left(q_{2}\right) \bar{D}_{p_{1}}\left(q_{12}\right) \bar{D}_{p_{2}}\left(q_{12}\right)}, \\
& \bar{D}_{p_{i}}\left(q_{j}\right)=\bar{q}_{j}^{2}+2\left(q_{j} \cdot p_{i}\right) .
\end{aligned}
$$

Note that the on-shell conditions $p_{1}^{2}=p_{2}^{2}=0$ are used at the integrand level. Thus, infrared virtual divergences get regulated by the $\mu^{2}$-deformed propagators, ${ }^{20}$ which generates powers of logarithms of $\mu^{2}$, upon integration. A particularly interesting situation is when the integral is also UV divergent. In this case it is easy to see that UV divergent scaleless $\ell$-loop FDR integrals vanish, as in DR. In fact, the only allowed external variable is a momentum $p$ such that $p^{2}=0$, whose fate is to appear in the numerator of $J_{\mathrm{F}, \ell}\left(q_{1}, \ldots, q_{\ell}\right)$ in Eq. (4) to improve the UV convergence of the original integrand. Therefore, $J_{\mathrm{F}, \ell}\left(q_{1}, \ldots, q_{\ell}\right)$ is entirely made of integrands proportional to positive powers of $\left(q_{i} \cdot p\right)$, which vanish, by Lorentz invariance, after integration. The simplest case is the fully massless one-loop 2-point scalar function

$B^{\mathrm{FDR}}\left(p^{2}=0,0,0\right)=\int\left[d^{4} q\right] \frac{1}{\bar{q}^{2}\left((q+p)^{2}-\mu^{2}\right)}$.

The FDR expansion of its integrand reads

$\frac{1}{\bar{q}^{2} \bar{D}_{p}}=\left[\frac{1}{\bar{q}^{4}}\right]-2 \frac{(q \cdot p)}{\bar{q}^{4} \bar{D}_{p}}$,

so that

$B^{\mathrm{FDR}}\left(p^{2}=0,0,0\right)=-2 \lim _{\mu \rightarrow 0} \int d^{4} q \frac{(q \cdot p)}{\bar{q}^{4} \bar{D}_{p}}=0$.

The same result is obtained by a direct computation

$$
\begin{aligned}
& B^{\mathrm{FDR}}\left(p^{2}, 0,0\right)=-i \pi^{2} \lim _{\mu \rightarrow 0} \int_{0}^{1} d x \\
& \times\left(\ln \left(\mu^{2}-p^{2} x(1-x)\right)-\ln \left(\mu^{2}\right)\right),
\end{aligned}
$$

from which it is manifest that, in the limit $p^{2} \rightarrow 0$, a cancelation occurs between two logarithms of UV and IR origin, respectively. ${ }^{21}$

$\overline{{ }^{20} \text { A different }} \mu^{2}$ can be used to regulate UV divergences ( $\mu_{\mathrm{UV}}^{2}$ ) and IR ones $\left(\mu_{\mathrm{IR}}^{2}\right)$. However, a common $\mu^{2}$ simplifies the calculation, as will be shown later. Since IR infinities are more easily understood in terms of $\mu_{\mathrm{IR}}^{2}>0$, it is convenient to choose $\mu_{\mathrm{UV}}^{2}=\mu_{\mathrm{IR}}^{2}=\mu^{2}>0$.

21 It is instructive to study the same case in DR, where $B^{\mathrm{DR}}\left(p^{2}, 0,0\right)=$ $\mu_{\mathrm{R}}^{-\epsilon} \int d^{n} q \frac{1}{q^{2}(q+p)^{2}}$. Now $B^{\mathrm{DR}}(0,0,0)$ vanishes because IR and UV poles in $\epsilon$ compensate. In fact, by introducing an arbitrary separation scale $M$, the two divergences can be disentangled

$$
\begin{aligned}
\frac{1}{(q+p)^{2}}= & \frac{1}{q^{2}-M^{2}}-\left(\frac{1}{q^{2}-M^{2}}-\frac{1}{(q+p)^{2}}\right)=\frac{1}{q^{2}-M^{2}} \\
& -\frac{M^{2}+2(q \cdot p)}{\left(q^{2}-M^{2}\right)(q+p)^{2}} .
\end{aligned}
$$

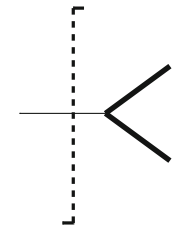

(a)

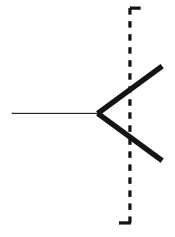

(b)
Fig. 4 Splitting regulated by massive (thick) unobserved particles. The one-particle cut in (a) contributes to the virtual part, the two-particle cut of (b) to the real radiation

In summary, IR divergent loop integrals are defined by taking the limit $\mu \rightarrow 0$ outside integration, after subtractingwhen necessary-UV divergent integrands. In order to preserve the cancelation of the IR logarithms in physical quantities, this definition should be accompanied by a consistent treatment of the infinities appearing in the real emission, which we discuss in the following.

Consider how the divergent $1 \rightarrow 2$ splitting is regulated in the loop integrals. The situation is depicted in Fig. 4a, where thick lines represent unobserved loop particles-whose propagator is made massive by the addition of $\mu^{2}$-and the cut line is an external observed massless particle. This is matched by the real radiation pattern of Fig. $4 \mathrm{~b}$, where thick lines are unobserved external particles merging into an internal observed massless one. In both situations unobserved particles get a mass $\mu$ and unitarity relates the two cases as follows:

$\frac{1}{q^{2}-\mu^{2}} \leftrightarrow \delta\left(q^{2}-\mu^{2}\right) \theta(q(0))$.

Therefore, would-be-massless external particles with momenta $p_{i}$ should be given a mass $\mu$. This is achieved by trading the original massless m-body phase space $d \Phi_{m}$ for a massive one, denoted by $d \bar{\Phi}_{m}$, in which

$p_{i}^{2} \rightarrow \bar{p}_{i}^{2}=\mu^{2}$.

Footnote 21 continued

Then the integrals $\left(\Delta=-\frac{2}{\epsilon}-\gamma_{E}-\ln \pi\right)$

$$
\begin{aligned}
I_{\mathrm{UV}} & =\mu_{\mathrm{R}}^{-\epsilon} \int d^{n} q \frac{1}{q^{2}\left(q^{2}-M^{2}\right)}=i \pi^{2}\left(\Delta-\ln \frac{M^{2}}{\mu_{\mathrm{R}}^{2}}+1\right), \\
I_{\mathrm{IR}} & =\mu_{\mathrm{R}}^{-\epsilon} \int d^{n} q \frac{M^{2}+2(q \cdot p)}{q^{2}\left(q^{2}-M^{2}\right)(q+p)^{2}}=I_{\mathrm{UV}}
\end{aligned}
$$

cancel each other. However, this argument has a potential problem, because it requires the cancelation of two analytic continuations, $I_{\mathrm{UV}}$ and $I_{\mathrm{IR}}$, originally defined in domains that do not overlap [48] $(\epsilon<0$ and $\epsilon>0$ ). Since no value of $\epsilon$ exists where they are defined simultaneously, it is not obvious whether their difference represents the original function $B^{\mathrm{DR}}(0,0,0)$. A possible mathematically consistent solution can be formulated in terms of modified Gaussian integrals in the $n$ dimensional Euclidean space [48]. In contrast, the FDR derivation in Eq. (57) is straightforward. 
In this way, singular configurations produce logarithms which cancel the IR dependence on $\mu^{2}$ of the virtual contribution. However, this strategy should be carried out without breaking gauge invariance. To illustrate the way to proceed we consider $m$-jet production at NNLO in $e^{+} e^{-}$annihilation. The building blocks of the calculation depend on the set of invariants

$$
\begin{aligned}
\left\{s_{i_{1} \div i_{m}}\right\} & \equiv\left\{s_{i_{1} i_{2}}, s_{i_{1} i_{2} i_{3}}, \ldots, s_{i_{1} \cdots i_{m}}\right\}, s_{i \cdots j} \\
& =\left(p_{i}+\cdots+p_{j}\right)^{2}, p_{i}^{2}=0 .
\end{aligned}
$$

They are:

- the Born contribution $d \sigma_{\mathrm{LO}}^{B}\left\{s_{i_{1} \div i_{m-1}}\right\}$,

- the virtual and real NLO corrections, $d \sigma_{\mathrm{NLO}}^{V}\left\{s_{i_{1}} \div i_{m-1}\right\}$ and $d \sigma_{\mathrm{NLO}}^{R}\left\{s_{i_{1} \div i_{m}}\right\}$,

- the NNLO two-loop part $d \sigma_{\mathrm{NNLO}}^{V, 2}\left\{s_{i_{1} \div i_{m-1}}\right\}$,

- the one-loop corrections to the NLO real radiation, $d \sigma_{\mathrm{NNLO}}^{V, 1}\left\{s_{i_{1} \div i_{m}}\right\}$,

- the double radiation $d \sigma_{\mathrm{NNLO}}^{R}\left\{s_{i_{1} \div i_{m+1}}\right\}$.

After $\alpha_{S}$ renormalization, they give a $m$-jet cross section accurate up to NNLO

$d \sigma=d \sigma_{\mathrm{LO}}+d \sigma_{\mathrm{NLO}}+d \sigma_{\mathrm{NNLO}}$,

where

$$
\begin{aligned}
d \sigma_{\mathrm{LO}}= & \int_{d \Phi_{m}} d \sigma_{\mathrm{LO}}^{B}\left\{s_{i_{1} \div i_{m-1}}\right\}, \\
d \sigma_{\mathrm{NLO}}= & \int_{d \Phi_{m}} d \sigma_{\mathrm{NLO}}^{V}\left\{s_{i_{1} \div i_{m-1}}\right\}+\int_{d \Phi_{m+1}} d \sigma_{\mathrm{NLO}}^{R}\left\{s_{i_{1} \div i_{m}}\right\} \\
d \sigma_{\mathrm{NNLO}}= & \int_{d \Phi_{m}} d \sigma_{\mathrm{NNLO}}^{V, 2}\left\{s_{i_{1} \div i_{m-1}}\right\}+\int_{d \Phi_{m+1}} d \sigma_{\mathrm{NNLO}}^{V, 1}\left\{s_{i_{1} \div i_{m}}\right\} \\
& +\int_{d \Phi_{m+2}} d \sigma_{\mathrm{NNLO}}^{R}\left\{s_{i_{1} \div i_{m+1}}\right\} .
\end{aligned}
$$

The integrands behave as

$$
\begin{aligned}
d \sigma\{\cdots\} & \sim \frac{1}{s_{i j}}, \quad \text { if } s_{i j} \rightarrow 0 \text { and } d \sigma\{\cdots\} \sim \frac{1}{s_{i j k}^{2}}, \\
\text { if } s_{i j k} & \rightarrow 0 ;
\end{aligned}
$$

therefore, the integrations over single- and double-unresolved massless phase spaces $\left(\int_{d \Phi_{m+1}}\right.$ and $\int_{d \Phi_{m+2}}$, respectively) generate logarithmic IR divergences which have to be regulated. In DR, the last two lines of Eq. (65) are interpreted as a limit to $\epsilon \rightarrow 0$ of integrals computed in $n=4+\epsilon$ dimensions. We instead define a mapping from massless to massive invariants as follows:

$$
\begin{aligned}
s_{i_{1} \cdots i_{m}} & \rightarrow \hat{s}_{i_{1} \cdots i_{m}} \equiv \sum_{k<l}^{m} \hat{s}_{i_{k} i_{l}}, \\
\hat{s}_{i j} & =\bar{s}_{i j}=\left(\bar{p}_{i}+\bar{p}_{j}\right)^{2}, \\
\bar{p}_{i}^{2} & =\mu^{2},
\end{aligned}
$$

and rewrite

$$
\begin{aligned}
d \sigma_{\mathrm{NLO}}= & \int_{d \Phi_{m}} d \sigma_{\mathrm{NLO}}^{V}\left\{s_{i_{1} \div i_{m-1}}\right\} \\
& +\lim _{\mu \rightarrow 0} \int_{d \bar{\Phi}_{m+1}} d \sigma_{\mathrm{NLO}}^{R}\left\{\hat{s}_{i_{1} \div i_{m}}\right\}, \\
d \sigma_{\mathrm{NNLO}}= & \int_{d \Phi_{m}} d \sigma_{\mathrm{NNLO}}^{V, 2}\left\{s_{i_{1} \div i_{m-1}}\right\} \\
& +\lim _{\mu \rightarrow 0} \int_{d \bar{\Phi}_{m+1}} d \sigma_{\mathrm{NNLO}}^{V, 1}\left\{\hat{s}_{i_{1} \div i_{m}}\right\}+\lim _{\mu \rightarrow 0} \int \\
& \quad \times d \sigma_{\mathrm{NNLO}}^{R}\left\{\hat{s}_{i_{1} \div i_{m+1}}\right\} W_{\mathrm{NNLO}}\left\{\hat{s}_{i_{1} i_{2} i_{3}}\right\}_{m+1}, \quad
\end{aligned}
$$

where $\mu$ is the same regulator used in the IR divergent loop integrals, and

$$
\begin{aligned}
& W_{\mathrm{NNLO}}\left\{\hat{s}_{i_{1} i_{2} i_{3}}\right\}_{m+1}=\prod_{i<j<k}^{m+1}\left(\frac{\hat{s}_{i j k}}{\bar{s}_{i j k}}\right)^{2}, \\
& \bar{s}_{i j k}=\left(\bar{p}_{i}+\bar{p}_{j}+\bar{p}_{k}\right)^{2} .
\end{aligned}
$$

The proof that Eq. (68) converges to the right results is simple. First note that the mapping in Eq. (67) preserves all formal properties of massless kinematics. For instance

$\hat{s}_{123}=\hat{s}_{12}+\hat{s}_{13}+\hat{s}_{23}$.

Thus, $d \sigma_{\mathrm{NLO}}^{R}, d \sigma_{\mathrm{NNLO}}^{V, 1}$ and $d \sigma_{\mathrm{NNLO}}^{R}$ are gauge invariant by construction. As for the NLO real emission, $\frac{1}{s_{i j k}^{2}}$ poles are always screened by the requirement of observing $m$ particles. Therefore, the only possible singular behavior is

$d \sigma_{\mathrm{NLO}}^{R}\left\{\hat{s}_{i_{1} \div i_{m}}\right\} \sim \frac{1}{\hat{s}_{i j}}=\frac{1}{\bar{s}_{i j}}=\frac{1}{\left(\bar{p}_{i}+\bar{p}_{j}\right)^{2}}$,

which, being the internal propagator massless, matches the virtual IR poles, as in Fig. 4b. In the NNLO case, $d \sigma_{\mathrm{NNLO}}^{R}\left\{\hat{s}_{i_{1} \div i_{m+1}}\right\}$ contains additional $\frac{1}{\hat{s}_{i j k}^{2}}$ poles, which no longer have the form of massless propagators. In fact, a spurious mass is generated by the gauge invariant mapping of Eq. (67):

$\hat{s}_{i j k}=\bar{s}_{i j k}+3 \mu^{2}=\left(\bar{p}_{i}+\bar{p}_{j}+\bar{p}_{k}\right)^{2}+3 \mu^{2}$.

To cure this, $d \sigma_{\mathrm{NNLO}}^{R}$ is multiplied by the weight factor in Eq. (69), which changes - in a gauge invariant way - any pole $\frac{1}{\hat{s}_{i j k}^{2}}$ to the correct value $\frac{1}{\hat{s}_{i j k}^{2}}$. The additional integrals, generated when each term in $W_{\mathrm{NNLO}}$ does not meet its corresponding pole, vanish in the limit $\mu \rightarrow 0$. This last property 
Fig. 5 Feynman diagrams contributing to the QCD corrections of the top-loop-mediated Higgs decay into two photons. The same diagrams with the electric charge flowing counterclockwise also contribute
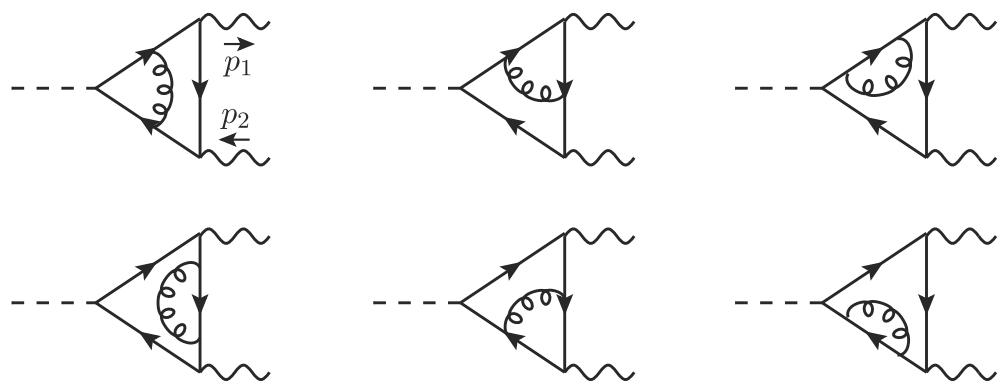

follows from the fact that the integral is at most logarithmically divergent. The reason why a pole cannot be changed by hand only in the terms where it appears is that the logarithmic behavior is reached only after gauge cancelations, which should not be altered. This can be easily understood with a toy model:

$\left.d \sigma_{\mathrm{NNLO}}^{R}\right|_{\text {toy }}=\frac{1}{\hat{s}_{12}^{2}}-\frac{\left(\hat{s}_{12}+\hat{s}_{13}+\hat{s}_{23}\right)^{2}}{\hat{s}_{12}^{2} \hat{s}_{123}^{2}}$.

The correct procedure gives

$\left.\lim _{\mu \rightarrow 0} \int_{d \bar{\Phi}_{m+2}} d \sigma_{\mathrm{NNLO}}^{R}\right|_{\text {toy }} W_{\mathrm{NNLO}}\left\{\hat{s}_{i_{1} i_{2} i_{3}}\right\}_{m+1}=0$,

while

$\lim _{\mu \rightarrow 0} \int_{d \bar{\Phi}_{m+2}}\left[\frac{1}{\hat{s}_{12}^{2}}-\frac{\left(\hat{s}_{12}+\hat{s}_{13}+\hat{s}_{23}\right)^{2}}{\hat{s}_{12}^{2} \bar{s}_{123}^{2}}\right] \neq 0$.

To summarize, IR infinities can be safely treated in four dimensions. An explicit one-loop example, involving both IR and UV infinities, can be found in [37]. Furthermore, the outlined strategy opens the possibility of a numerical treatment of NNLO calculations similar to the phase-space slicing method at NLO [49]. The advantage is that all singularities are automatically expressed in terms of powers of a logarithmic regulator- $\ln \mu$-with no need of subtracting $1 / \epsilon$ poles. An investigation of the numerical performance of such a strategy is outside the scope of this work, although it is currently under study. We think that it is a promising one because, owing to the four-dimensionality of the calculation, we envisage that the bulk of the cancelations can be easily arranged to happen at the integrand level.

\section{$3 \mathrm{H} \rightarrow \gamma \gamma$ at two loops}

The diagrams contributing to the QCD corrections of the toploop-mediated Higgs decay into two photons are depicted in Fig. 5.

The amplitude reads

$\mathcal{M}=\mathcal{M}^{\alpha \beta} \varepsilon_{\alpha}\left(p_{1}\right) \varepsilon_{\beta}^{*}\left(p_{2}\right)$, where $p_{1}$ and $-p_{2}$ are the momenta of the outgoing photons. One has

$\mathcal{M}^{\alpha \beta}=\frac{1}{(2 \pi)^{2}} \frac{\alpha}{\pi} \frac{T^{\alpha \beta}}{v} \frac{4}{3} \eta \mathcal{F}(\eta)$

with $v$ being the vev of the Higgs boson and

$$
\begin{gathered}
\eta=\frac{4 m^{2}}{s}, \quad m=m_{\text {top }}, \quad s=\left(p_{1}-p_{2}\right)^{2}=M_{H}^{2}, \\
T^{\alpha \beta}=p_{2}^{\alpha} p_{1}^{\beta}+\frac{s}{2} g^{\alpha \beta} .
\end{gathered}
$$

$\mathcal{M}$ is well defined in the limit $m \rightarrow \infty$ we are interested in. This means that, order by order, the form factor $\mathcal{F}(\eta)$ can be written as

$\mathcal{F}(\eta)=\mathcal{F}_{0}+\frac{\mathcal{F}_{1}}{\eta}+\frac{\mathcal{F}_{2}}{\eta^{2}}+\cdots$

with

$\mathcal{F}_{0}=0$.

By inserting Eq. (79) into the expansion in $\alpha_{S}$ of $\mathcal{F}(\eta)$, one obtains, up to two loops and neglecting $\mathcal{O}\left(\frac{1}{\eta^{2}}\right)$ terms,

$$
\begin{aligned}
\mathcal{F}(\eta) & =\mathcal{F}^{(1)}(\eta)-i \frac{\alpha_{S}}{3 \pi^{3}} \mathcal{F}^{(2)}(\eta) \\
& \equiv\left(\mathcal{F}_{0}^{(1)}+\frac{\mathcal{F}_{1}^{(1)}}{\eta}\right)-i \frac{\alpha_{S}}{3 \pi^{3}}\left(\mathcal{F}_{0}^{(2)}+\frac{\mathcal{F}_{1}^{(2)}}{\eta}\right)
\end{aligned}
$$

At one loop $\mathcal{F}_{0}^{(1)}=0$ and (see, for example, [38])

$\mathcal{F}_{1}^{(1)}=\frac{4 i \pi^{2}}{3}$.

In this section, we re-derive ${ }^{22}$-within the FDR framework - the known result [50]

$\mathcal{F}_{0}^{(2)}=0$

$\mathcal{F}_{1}^{(2)}=4 \pi^{4}$,

which implies that the QCD corrections factorize the oneloop amplitude

$$
\mathcal{M}=\mathcal{M}^{(1)}\left(1-\frac{\alpha_{S}}{\pi}\right)+\mathcal{O}\left(\alpha_{S}^{2}\right)+\mathcal{O}\left(\frac{1}{\eta}\right) .
$$

$\overline{22}$ We use the Feynman rules of Appendix B. 


\subsection{The building blocks}

Since we are working in the large top mass limit, denominators can be expanded as follows:

$$
\begin{aligned}
\frac{1}{\left(q_{i}+p_{j}\right)^{2}-m^{2}} & =\frac{1}{q_{i}^{2}-m^{2}}\left(1-\frac{2\left(q_{i} \cdot p_{j}\right)+p_{j}^{2}}{\left(q_{i}+p_{j}\right)^{2}-m^{2}}\right) \\
& =\frac{1}{q_{i}^{2}-m^{2}}\left(1-\frac{2\left(q_{i} \cdot p_{j}\right)}{q_{i}^{2}-m^{2}}+\cdots\right),
\end{aligned}
$$

where the on-shell condition $p_{j}^{2}=0$ for the photons has been used. An expansion to the second order, as the one above, is sufficient to the level of accuracy we are interested in, i.e. $\mathcal{O}(1 / \eta)$. All external momenta can then be neglected and the top mass is the only relevant scale. As a consequence, we only have to deal with vacuum integrals.

After canceling between numerator and denominator the $\bar{q}_{1}^{2}, \bar{q}_{2}^{2}, \bar{q}_{12}^{2}$ terms generated by the Feynman rules, ${ }^{23}$ tensor integrals up to rank 4 contribute to the amplitude. Because there is no dependence on external momenta, odd rank integrals vanish and the tensor reduction gives

$q_{i}^{\alpha} q_{j}^{\beta} \rightarrow \frac{\left(q_{i} \cdot q_{j}\right)}{4} g^{\alpha \beta}$,

$q^{\alpha} q^{\beta} q^{\rho} q^{\sigma} \rightarrow \frac{q^{4}}{24} g^{\alpha \beta \rho \sigma}$ at one loop,

$q_{a}^{\alpha} q_{b}^{\beta} q_{r}^{\rho} q_{s}^{\sigma} \rightarrow \frac{1}{72}\left(A_{a b r s}^{\alpha \beta \rho \sigma}+A_{a r b s}^{\alpha \rho \beta \sigma}+A_{a s b r}^{\alpha \sigma \beta \rho}\right)$ at two loops,

where $g^{\alpha \beta \rho \sigma}=g^{\alpha \beta} g^{\rho \sigma}+g^{\alpha \rho} g^{\beta \sigma}+g^{\alpha \sigma} g^{\beta \rho}$, and

$$
\begin{aligned}
A_{a b r s}^{\alpha \beta \rho \sigma}= & \left(5\left(q_{a} \cdot q_{b}\right)\left(q_{r} \cdot q_{s}\right)-\left(q_{a} \cdot q_{r}\right)\left(q_{b} \cdot q_{s}\right)\right. \\
& \left.-\left(q_{a} \cdot q_{s}\right)\left(q_{b} \cdot q_{r}\right)\right) g^{\alpha \beta} g^{\rho \sigma} .
\end{aligned}
$$

Denominators can then be reconstructed by rewriting

$q_{1}^{2}=\bar{q}_{1}^{2}+\left.\mu^{2}\right|_{1}, \quad q_{2}^{2}=\bar{q}_{2}^{2}+\left.\mu^{2}\right|_{2}$,

$2\left(q_{1} \cdot q_{2}\right)=\bar{q}_{12}^{2}-\bar{q}_{1}^{2}-\bar{q}_{2}^{2}+\left.\mu^{2}\right|_{12}-\left.\mu^{2}\right|_{1}-\left.\mu^{2}\right|_{2}$.

During this tensor decomposition, the $\left.\mu^{2}\right|_{1},\left.\mu^{2}\right|_{2},\left.\mu^{2}\right|_{12}$ terms are kept only when they generate a non-zero contribution. This means that they should be power-counted as the corresponding squared loop momenta, and contribute only if the integral is divergent. The final result can then be completely expressed in terms of scalar two-loop integrals, products of two one-loop integrals and extra integrals containing

${ }^{23}$ Remember the discussion at the beginning of Sect. 2.4. $\left.\mu^{2}\right|_{j}(j=1,2,12)$. For convenience, we introduce the notation

$$
\begin{aligned}
& {[\alpha m]=\int \frac{\left[\mathrm{d}^{4} q\right]}{\left(\bar{q}^{2}-m^{2}\right)^{\alpha}},} \\
& {\left[\alpha m_{1} \mid \beta m_{2}\right]=\int \frac{\left[\mathrm{d}^{4} q_{1}\right]}{\left(\bar{q}_{1}^{2}-m_{1}^{2}\right)^{\alpha}} \times \int \frac{\left[\mathrm{d}^{4} q_{2}\right]}{\left(\bar{q}_{2}^{2}-m_{2}^{2}\right)^{\beta}},} \\
& {\left[\alpha m_{1}\left|\beta m_{2}\right| 0\right]=\int \frac{\left[\mathrm{d}^{4} q_{1}\right]\left[\mathrm{d}^{4} q_{2}\right]}{\left(\bar{q}_{1}^{2}-m_{1}^{2}\right)^{\alpha}\left(\bar{q}_{2}^{2}-m_{2}^{2}\right)^{\beta} \bar{q}_{12}^{2}},}
\end{aligned}
$$

and

$$
\begin{aligned}
& {[\alpha m]\left(\mu^{2}\right)=\int \frac{\left[\mathrm{d}^{4} q\right] \mu^{2}}{\left(\bar{q}^{2}-m^{2}\right)^{\alpha}},} \\
& {\left[\alpha m_{1} \mid \beta m_{2}\right]\left(\left.\mu^{2}\right|_{1}\right)=\int \frac{\left.\left[\mathrm{d}^{4} q_{1}\right] \mu^{2}\right|_{1}}{\left(\bar{q}_{1}^{2}-m_{1}^{2}\right)^{\alpha}} \times \int \frac{\left[\mathrm{d}^{4} q_{2}\right]}{\left(\bar{q}_{2}^{2}-m_{2}^{2}\right)^{\beta}},} \\
& {\left[\alpha m_{1}\left|\beta m_{2}\right| 0\right]\left(\left.\mu^{2}\right|_{j}\right)=\int \frac{\left.\left[\mathrm{d}^{4} q_{1}\right]\left[\mathrm{d}^{4} q_{2}\right] \mu^{2}\right|_{j}}{\left(\bar{q}_{1}^{2}-m_{1}^{2}\right)^{\alpha}\left(\bar{q}_{2}^{2}-m_{2}^{2}\right)^{\beta} \bar{q}_{12}^{2}} .}
\end{aligned}
$$

The one-loop and factorizable integrals of Eqs. (89) and (90) can be computed as derivatives of the quadratically divergent one-loop tadpole [30]

$$
\begin{aligned}
& \int\left[\mathrm{d}^{4} q\right] \frac{1}{\left(\bar{q}^{2}-m^{2}\right)^{\alpha}}=\frac{1}{\Gamma(\alpha)} \frac{\mathrm{d}^{\alpha-1}}{\mathrm{~d}\left(m^{2}\right)^{\alpha-1}} \int\left[\mathrm{d}^{4} q\right] \frac{1}{\left(\bar{q}^{2}-m^{2}\right)} \\
& \int\left[\mathrm{d}^{4} q\right] \frac{1}{\left(\bar{q}^{2}-m^{2}\right)}=-i \pi^{2} m^{2}\left(\log \frac{m^{2}}{\mu_{\mathrm{R}}^{2}}-1\right)
\end{aligned}
$$

while those in Eq. (91) are obtained by deriving with respect to the mass parameters the basic integral

$\left[2 m_{1}\left|m_{2}\right| 0\right]$

computed in Appendix $\mathrm{D}^{24}$

$$
\begin{aligned}
{\left[\alpha m_{1}\left|\beta m_{2}\right| 0\right]=} & \frac{1}{\Gamma(\alpha) \Gamma(\beta)} \frac{\mathrm{d}^{\alpha-2}}{\mathrm{~d}\left(m_{1}^{2}\right)^{\alpha-2}} \frac{\mathrm{d}^{\beta-1}}{\mathrm{~d}\left(m_{2}^{2}\right)^{\beta-1}} \\
& \times\left[2 m_{1}\left|m_{2}\right| 0\right] .
\end{aligned}
$$

All extra integrals relevant for our calculation can be expressed in terms of three fundamental objects

$\mu^{2} \int d^{4} q \frac{1}{\bar{q}^{6}}=-\frac{i \pi^{2}}{2}$,

$\mu^{2} \int d^{4} q_{1} d^{4} q_{2} \frac{q_{1}^{2}+2\left(q_{1} \cdot q_{2}\right)}{\bar{q}_{1}^{6} \bar{q}_{2}^{4} \bar{q}_{12}^{2}}=-\pi^{4}\left(\frac{2}{3} f+\frac{1}{2}\right)$,

$\mu^{2} \int d^{4} q_{1} d^{4} q_{2} \frac{1}{\bar{q}_{1}^{4} \bar{q}_{2}^{4} \bar{q}_{12}^{2}}=-\frac{2 \pi^{4}}{3} f$

\footnotetext{
24 This implies that for each of the diagrams in Fig. 5 the routing of the momenta is chosen such that the gluon line gets the momentum $q_{12}$. This is allowed due to the shift invariance properties of the FDR integration.
} 
with $f$ given in Eq. (130). We need

$[3 m|m| 0]\left(\left.\mu^{2}\right|_{1}\right)$,

derived in footnote 16 , and

$\int\left[\mathrm{d}^{4} q\right] \frac{\mu^{2}}{\left(\bar{q}^{2}-m^{2}\right)^{\alpha}}=\frac{1}{\Gamma(\alpha)} \frac{\mathrm{d}^{\alpha-1}}{\mathrm{~d}\left(m^{2}\right)^{\alpha-1}} \int\left[\mathrm{d}^{4} q\right] \frac{\mu^{2}}{\left(\bar{q}^{2}-m^{2}\right)}$,

$\left[\alpha m_{1}\left|\beta m_{2}\right| 0\right]\left(\left.\mu^{2}\right|_{j}\right)$

$$
=\frac{1}{\Gamma(\alpha) \Gamma(\beta)} \frac{\mathrm{d}^{\alpha-2}}{\mathrm{~d}\left(m_{1}^{2}\right)^{\alpha-2}} \frac{\mathrm{d}^{\beta-2}}{\mathrm{~d}\left(m_{2}^{2}\right)^{\beta-2}}\left[2 m_{1}\left|2 m_{2}\right| 0\right]\left(\left.\mu^{2}\right|_{j}\right)
$$

with

$$
\begin{aligned}
& \int\left[\mathrm{d}^{4} q\right] \frac{\mu^{2}}{\left(\bar{q}^{2}-m^{2}\right)}=-m^{4} \lim _{\mu \rightarrow 0} \mu^{2} \int d^{4} q \frac{1}{\bar{q}^{6}}, \\
& {\left[2 m_{1}\left|2 m_{2}\right| 0\right]\left(\left.\mu^{2}\right|_{1}\right)=-\lim _{\mu \rightarrow 0} \mu^{2}\left\{\int \frac{d^{4} q_{1} d^{4} q_{2}}{\bar{q}_{1}^{4} \bar{q}_{2}^{4} \bar{q}_{12}^{2}}\right.} \\
& \left.-i \pi^{2} \ln \frac{m_{2}^{2}}{\mu^{2}} \int \frac{d^{4} q}{\bar{q}^{6}}\right\} \\
& {\left[2 m_{1}\left|2 m_{2}\right| 0\right]\left(\left.\mu^{2}\right|_{2}\right)=-\lim _{\mu \rightarrow 0} \mu^{2}\left\{\int \frac{d^{4} q_{1} d^{4} q_{2}}{\bar{q}_{1}^{4} \bar{q}_{2}^{4} \bar{q}_{12}^{2}}\right.} \\
& \left.-i \pi^{2} \ln \frac{m_{1}^{2}}{\mu^{2}} \int \frac{d^{4} q}{\bar{q}^{6}}\right\} \\
& {\left[2 m_{1}\left|2 m_{2}\right| 0\right]\left(\left.\mu^{2}\right|_{12}\right)=-\lim _{\mu \rightarrow 0} \mu^{2}\left\{\int \frac{d^{4} q_{1} d^{4} q_{2}}{\bar{q}_{1}^{4} \bar{q}_{2}^{4} \bar{q}_{12}^{2}}\right.} \\
& \left.-i \pi^{2}\left(\ln \frac{m_{1}^{2}}{\mu^{2}}+\ln \frac{m_{2}^{2}}{\mu^{2}}\right) \int \frac{d^{4} q}{\bar{q}^{6}}\right\} \text {. }
\end{aligned}
$$

The first of Eqs. (100) is computed indirectly from the FDR expansion

$$
\begin{aligned}
\frac{q^{\alpha} q^{\beta}}{\left(\bar{q}^{2}-m^{2}\right)}= & q^{\alpha} q^{\beta}\left\{\left[\frac{1}{\bar{q}^{2}}\right]+m^{2}\left[\frac{1}{\bar{q}^{4}}\right]\right. \\
& \left.+m^{4}\left[\frac{1}{\bar{q}^{6}}\right]+m^{6}\left[\frac{1}{\bar{q}^{6}\left(\bar{q}^{2}-m^{2}\right)}\right]\right\},
\end{aligned}
$$

while Eqs. (127) and (128) give the other three equalities.

\subsection{The result}

By summing all Feynman diagrams and performing the tensor reduction we end up with

$$
\begin{aligned}
\mathcal{F}_{0}^{(2)}= & -2[2 m \mid 2 m]+4[3 m \mid m]-4 m^{2}[3 m \mid 2 m] \\
& +12 m^{2}[4 m \mid m]+4[2 m|m| 0] \\
& +12 m^{2}(2[3 m|m| 0]+[2 m|2 m| 0]) \\
& +24 m^{4}([4 m|m| 0]+[3 m|2 m| 0]) \\
& +4[3 m \mid 2 m]\left(\left.\mu^{2}\right|_{1}\right) \\
& +8[3 m|m| 0]\left(\left.\mu^{2}\right|_{1}\right)+4[2 m|2 m| 0]\left(\left.\mu^{2}\right|_{1}\right) \\
& -2[2 m|2 m| 0]\left(\left.\mu^{2}\right|_{12}\right) \\
& +8 m^{2}\left([3 m|2 m| 0]\left(\left.\mu^{2}\right|_{2}\right)\right. \\
& \left.-[3 m|2 m| 0]\left(\left.\mu^{2}\right|_{12}\right)\right),
\end{aligned}
$$

and

$$
\begin{aligned}
\mathcal{F}_{1}^{(2)}= & +\frac{176}{9} m^{2}[3 m \mid 2 m]-\frac{56}{3} m^{2}[4 m \mid m] \\
& -4 m^{4}\left(\frac{10}{9}[3 m \mid 3 m]-\frac{10}{3}[4 m \mid 2 m]\right. \\
& \left.+\frac{16}{3}[5 m \mid m]\right)+4 m^{6}\left(\frac{10}{3}[4 m \mid 3 m]\right. \\
& \left.+4[5 m \mid 2 m]-\frac{20}{3}[6 m \mid m]\right) \\
& -\frac{320}{9} m^{2}[3 m|m| 0]-\frac{136}{9} m^{2}[2 m|2 m| 0] \\
& -\frac{176}{3} m^{4}([4 m|m| 0]+[3 m|2 m| 0]) \\
& -\frac{224}{3} m^{6}([5 m|m| 0]+[4 m|2 m| 0] \\
& \left.+\frac{1}{2}[3 m|3 m| 0]\right) \\
& -\frac{160}{3} m^{8}([6 m|m| 0]+[5 m|2 m| 0] \\
& +[4 m|3 m| 0]) \\
& -8 m^{2}[3 m \mid 3 m]\left(\left.\mu^{2}\right|_{1}\right)-8 m^{4}[3 m \mid 4 m]\left(\left.\mu^{2}\right|_{1}\right) \\
& +\frac{64}{9} m^{2}[3 m|2 m| 0]\left(\left.\mu^{2}\right|_{2}\right) \\
& +\frac{80}{9} m^{2}[3 m|2 m| 0]\left(\left.\mu^{2}\right|_{12}\right) \\
& -16 m^{4}\left([4 m|2 m| 0]\left(\left.\mu^{2}\right|_{2}\right)\right. \\
& \left.-[4 m|2 m| 0]\left(\left.\mu^{2}\right|_{12}\right)\right) \\
& -\frac{64}{3} m^{6}\left([5 m|2 m| 0]\left(\left.\mu^{2}\right|_{2}\right)\right. \\
& \left.-\left[5 m^{2}|2 m| 0\right]\left(\mu^{2} \mid 12\right)\right) \\
& -[3 m) \\
&
\end{aligned}
$$

The final result in Eq. (83) follows by inserting the expressions of the scalar and extra integrals computed in Sect. 3.1.

A few remarks are in order. At two loops the one-toone correspondence between DR and FDR is lost and it is no longer true that FDR integrals are obtained from DR ones after subtracting poles (and universal constants). For 
example, if we were to interpret the integrals appearing in Eq. (102) as dimensionally regulated ones, we would not get zero and a $1 / \epsilon$ pole would even remain! Differences already start at the level of the basic two-loop scalar integral. The DR counterpart of Eq. (129) reads [51]

$$
\begin{aligned}
& \mu_{\mathrm{R}}^{-2 \epsilon} \int d^{n} q_{1} d^{n} q_{2} \frac{1}{\left(q_{1}^{2}-m_{1}^{2}\right)^{2}\left(q_{2}^{2}-m_{2}^{2}\right) q_{12}^{2}} \\
& =\pi^{4}\left\{-\mathrm{Li}_{2}\left(1-\frac{m_{2}^{2}}{m_{1}^{2}}\right)-\ln ^{2} \frac{\mu_{\mathrm{R}}^{2}}{m_{1}^{2}}-\ln \frac{\mu_{\mathrm{R}}^{2}}{m_{1}^{2}}+\text { constant }\right\},
\end{aligned}
$$

with a different coefficient in front of the $\ln ^{2}$. This can be understood because two different mechanisms to preserve gauge invariance are used by DR and FDR, the latter avoiding an order-by-order renormalization. Another advantage of FDR is that the same formulas for the scalar one-loop functions can be used also when they combine to form a factorizable two-loop integral. Differently stated, Eq. (90) is simply the product of two integrals of the kind given in Eq. (89). This does not happen in DR, where terms of $\mathcal{O}(\epsilon)$ must be added to the one-loop functions appearing in a two-loop calculation. Note also that there is no need of renormalizing $\mathcal{F}_{0}^{(2)}$ and $\mathcal{F}_{1}^{(2)}$. This directly follows from the discussion in Sect. 2.5. FDR renormalization amounts to the mere operation of fixing results in terms of physical quantities, and since the top mass disappears due to the limit $m_{\text {top }} \rightarrow \infty$, no fixing is needed. This is not the case when using DR, where renormalization is required in order to compensate spurious $\epsilon / \epsilon$ constants generated in the limit $n \rightarrow 4$. The situation is analyzed in the next subsection.

\subsection{Renormalization}

Here we demonstrate that if we insist with an order-by-order renormalization we obtain a vanishing contribution to $\mathcal{F}_{0}^{(2)}$ and $\mathcal{F}_{1}^{(2)}$. At $\mathcal{O}\left(\alpha_{S}\right)$ the bare $\left(m_{0}\right)$ and physical $(m)$ top masses satisfy the relation

$m_{0}=m+\delta m, \quad \delta m=\Sigma(m)$,

where $\Sigma(\not p)$ is the top self-energy depicted in Fig. 6 and

$\Sigma(m)=m \frac{\alpha_{S}}{3 \pi}\left(3 \ln \frac{m^{2}}{\mu_{\mathrm{R}}^{2}}-5\right)$.

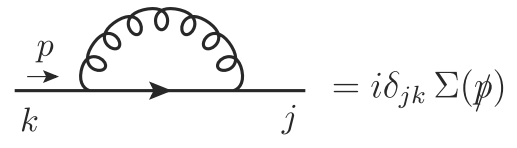

Fig. 6 Top self-energy at $\mathcal{O}\left(\alpha_{S}\right)$

This gives the one-loop counterterms and diagrams of Fig. 7, which generate a contribution to $\mathcal{F}_{0}^{(2)}$ and $\mathcal{F}_{1}^{(2)}$ proportional to

$\mathcal{F}_{0, c t}^{(2)}=i \delta m C_{0, c t} \quad$ and $\quad \mathcal{F}_{1, c t}^{(2)}=i \delta m C_{1, c t}$.

One computes

$$
\begin{aligned}
C_{0, c t}= & 8 m^{2}[3 m]+12 m^{4}[4 m]+4[3 m]\left(\mu^{2}\right)=0, \\
C_{1, c t}= & -\frac{16 m^{2}}{3}\left([3 m]+4 m^{2}[4 m]+5 m^{4}[5 m]\right. \\
& \left.+5 m^{6}[6 m]\right)=0 .
\end{aligned}
$$

Therefore renormalization does not have any effect.

It is worth mentioning that in DR

$\left.C_{0, \mathrm{ct}}\right|_{\mathrm{DR}}=0$ and $\left.C_{1, \mathrm{ct}}\right|_{\mathrm{DR}}=\mathcal{O}(\epsilon)$,

so that $\left.C_{1, \mathrm{ct}}\right|_{\mathrm{DR}}$ contributes to the amplitude when multiplied against the $1 / \epsilon$ pole contained in $\left.\delta m\right|_{\mathrm{DR}}$ (the DR counterpart of $\delta m^{25}$ ), and renormalization is necessary.

\section{Conclusions}

We have presented the first two-loop calculation ever performed in FDR. The $\mathcal{O}\left(\alpha_{S}\right)$ corrections to the $H \rightarrow \gamma \gamma$ amplitude - mediated by an infinitely heavy top loop—have been computed in a fully four-dimensional fashion. This example has allowed us to show that FDR is an approach to loop calculations in which

- gauge invariance is preserved;

- order-by-order renormalization is avoided;

- a finite renormalization is only needed to fix the parameters of the theory in terms of experimental observables;

- $\ell$-loop integrals are directly re-usable in $(\ell+1)$-loop calculations, with no need of further expanding in $\epsilon$.

In addition, we have described how infrared divergences can be dealt with within the same four-dimensional framework used to cope with the ultraviolet infinities.

We have also demonstrated that DR and FDR are not related in a direct way-beyond one loop-since FDR integrals cannot be interpreted any longer as DR ones devoid of

\footnotetext{
$\left.{ }^{25} \delta m\right|_{\mathrm{DR}}$ in dimensional reduction is obtained from Eq. (106) through the replacement $\ln \mu_{\mathrm{R}}^{2} \rightarrow \ln \mu_{\mathrm{R}}^{2}+\Delta$, with $\Delta$ given in footnote 21 .
} 
Fig. 7 One-loop counterterms and diagrams generated by Eq. (105)
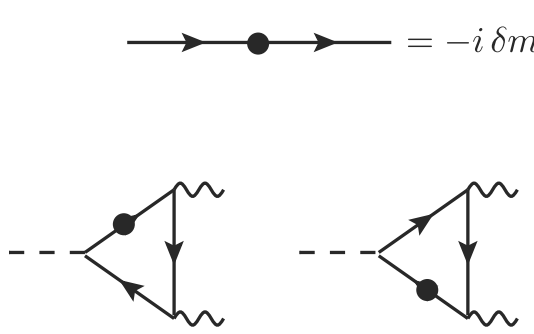

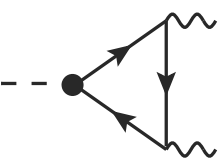

the pole part. Due to its four-dimensionality we envisage a great potential of FDR in further simplifying NNLO computations. More investigation is needed in this direction, which we plan to undertake in the near future.

Acknowledgments This work was supported by the European Commission through contracts ERC-2011-AdG No 291377 (LHCtheory) and PITN-GA-2012-316704 (HIGGSTOOLS). We also thank the support of the MICINN project FPA2011-22398 (LHC@NLO) and the Junta de Andalucía project P10-FQM-6552.

Open Access This article is distributed under the terms of the Creative Commons Attribution License which permits any use, distribution, and reproduction in any medium, provided the original author(s) and the source are credited.

Funded by $\mathrm{SCOAP}^{3}$ / License Version CC BY 4.0.

\section{Appendix A: FDR and shift invariance}

In this appendix we demonstrate that, for positive integers $\alpha$, $\beta, \gamma$, and $\delta$,

$$
\begin{aligned}
& \int\left[d^{4} q\right] \frac{1}{\left(q^{2}-m^{2}-\mu^{2}\right)^{\alpha}} \\
& =\int\left[d^{4} q\right] \frac{1}{\left((q+p)^{2}-m^{2}-\mu^{2}\right)^{\alpha}}
\end{aligned}
$$

and

$$
\begin{aligned}
& \int \frac{\left[d^{4} q_{1}\right]\left[d^{4} q_{2}\right]}{\left(q_{1}^{2}-m_{1}^{2}-\mu^{2}\right)^{\beta}\left(q_{2}^{2}-m_{2}^{2}-\mu^{2}\right)^{\gamma}\left(q_{12}^{2}-m_{12}^{2}-\mu^{2}\right)^{\delta}} \\
& =\int \frac{\left[d^{4} q_{1}\right]\left[d^{4} q_{2}\right]}{\left(\left(q_{1}+p_{1}\right)^{2}-m_{1}^{2}-\mu^{2}\right)^{\beta}\left(\left(q_{2}+p_{2}\right)^{2}-m_{2}^{2}-\mu^{2}\right)^{\gamma}\left(\left(q_{12}+p_{12}\right)^{2}-m_{12}^{2}-\mu^{2}\right)^{\delta}},
\end{aligned}
$$

In all the other cases Eqs. (110) and (111) coincide with finite integrals, for which shift invariance trivially holds.

We start proving Eq. (110) with $\alpha=1$. By using the shorthand notation

$\bar{D}=\left(q^{2}-m^{2}-\mu^{2}\right), \quad \bar{S}=(q+p)^{2}-m^{2}-\mu^{2}$,

one writes the FDR expansions of the two sides of the equation as

$$
\begin{aligned}
\frac{1}{\bar{D}}= & {\left[\frac{1}{\bar{q}^{2}}\right]+\left[\frac{m^{2}}{\bar{q}^{4}}\right]+J_{\mathrm{F}, 1}(q), } \\
\frac{1}{\bar{S}}= & {\left[\frac{1}{\bar{q}^{2}}\right]+\left[\frac{m^{2}-p^{2}-2(q \cdot p)}{\bar{q}^{4}}\right]+4\left[\frac{(q \cdot p)^{2}}{\bar{q}^{6}}\right] } \\
& +J_{\mathrm{F}, 1}^{\prime}(q) .
\end{aligned}
$$

Then

$$
\begin{aligned}
\int\left[d^{4} q\right] \frac{1}{\bar{D}}= & \lim _{\mu \rightarrow 0} \mu_{\mathrm{R}}^{-\epsilon}\left(\int d^{n} q \frac{1}{\bar{D}}-\int d^{n} q \frac{m^{2}}{\bar{q}^{4}}\right. \\
& \left.-\int d^{n} q \frac{1}{\bar{q}^{2}}\right) \\
= & \lim _{\mu \rightarrow 0} \mu_{\mathrm{R}}^{-\epsilon}\left(\int d^{n} q \frac{1}{\bar{S}}-\int d^{n} q \frac{m^{2}}{\bar{q}^{4}}\right. \\
& \left.-\int d^{n} q \frac{1}{(q+p)^{2}-\mu^{2}}\right),
\end{aligned}
$$

where the shift invariance of the dimensionally regulated integrals over $1 / \bar{D}$ and $1 / \bar{q}^{2}$ has been used. By expanding the last integrand one obtains where $q_{12}=q_{1}+q_{2}$ and $p_{12}=p_{1}+p_{2}$. Since integrals of polynomials in the integration variables vanish, the divergent parts of any one- or two-loop FDR integral can be writtenafter expanding in the external momenta-in terms of the four cases

$\alpha=1, \quad \alpha=2, \quad \beta=\gamma=\delta=1$, and

$\beta=\gamma=1$ with $\delta>1$.

$$
\begin{aligned}
\frac{1}{(q+p)^{2}-\mu^{2}}-\frac{1}{\bar{q}^{2}}= & -\frac{p^{2}+2(q \cdot p)}{\bar{q}^{4}}+4 \frac{(q \cdot p)^{2}}{\bar{q}^{6}} \\
& +\mathcal{O}\left(p^{3}\right)
\end{aligned}
$$

Since the 1.h.s. of Eq. (116) vanishes upon integration at any order in $p$, the same happens for the combination

$-\frac{p^{2}+2(q \cdot p)}{\bar{q}^{4}}+4 \frac{(q \cdot p)^{2}}{\bar{q}^{6}}$. 
The last integral in Eq. (115) can then be rewritten as

$$
\begin{aligned}
& \mu_{\mathrm{R}}^{-\epsilon} \int d^{n} q \frac{1}{(q+p)^{2}-\mu^{2}}=\mu_{\mathrm{R}}^{-\epsilon} \int d^{n} q \\
& \quad \times\left(\frac{1}{\bar{q}^{2}}-\frac{p^{2}+2(q \cdot p)}{\bar{q}^{4}}+4 \frac{(q \cdot p)^{2}}{\bar{q}^{6}}\right),
\end{aligned}
$$

so that

$$
\int\left[d^{4} q\right] \frac{1}{\bar{D}}=\lim _{\mu \rightarrow 0} \int d^{4} q J_{\mathrm{F}, 1}^{\prime}(q)=\int\left[d^{4} q\right] \frac{1}{\bar{S}},
$$

which proves Eq. (110) with $\alpha=1$. The case $\alpha=2$ is proven by taking the derivative of Eq. (119) with respect to $m^{2}$.

We now deal with the case $\beta=\gamma=\delta=1$. The FDR expansion of the 1.h.s. of Eq. (111) is given by Eq. (8). As for the r.h.s., we introduce

$$
\begin{gathered}
\bar{S}_{i}=\left(q_{i}+p_{i}\right)^{2}-m_{i}^{2}-\mu^{2} \text { and } \\
N_{i}=m_{i}^{2}-p_{i}^{2}-2\left(q_{i} \cdot p_{i}\right),
\end{gathered}
$$

in terms of which the expansion reads

$$
\begin{aligned}
& \frac{1}{\bar{S}_{1} \bar{S}_{2} \bar{S}_{12}}=\left[\frac{1}{\bar{q}_{1}^{2} \bar{q}_{2}^{2} \bar{q}_{12}^{2}}\right]+\left[\frac{N_{1}}{\bar{q}_{1}^{4} \bar{q}_{2}^{2} \bar{q}_{12}^{2}}\right]+\left[\frac{N_{2}}{\bar{q}_{1}^{2} \bar{q}_{2}^{4} \bar{q}_{12}^{2}}\right] \\
& +\left[\frac{N_{12}}{\bar{q}_{1}^{2} \bar{q}_{2}^{2} \bar{q}_{12}^{4}}\right]+4\left[\frac{\left(q_{1} \cdot p_{1}\right)^{2}}{\bar{q}_{1}^{6} \bar{q}_{2}^{2} \bar{q}_{12}^{2}}\right]+4\left[\frac{\left(q_{2} \cdot p_{2}\right)^{2}}{\bar{q}_{1}^{2} \bar{q}_{2}^{6} \bar{q}_{12}^{2}}\right] \\
& +4\left[\frac{\left(q_{12} \cdot p_{12}\right)^{2}}{\bar{q}_{1}^{2} \bar{q}_{2}^{2} \bar{q}_{12}^{6}}\right]+4\left[\frac{\left(q_{1} \cdot p_{1}\right)\left(q_{2} \cdot p_{2}\right)}{\bar{q}_{1}^{4} \bar{q}_{2}^{4} \bar{q}_{12}^{2}}\right] \\
& +4\left[\frac{\left(q_{1} \cdot p_{1}\right)\left(q_{12} \cdot p_{12}\right)}{\bar{q}_{1}^{4} \bar{q}_{2}^{2} \bar{q}_{12}^{4}}\right]+4\left[\frac{\left(q_{2} \cdot p_{2}\right)\left(q_{12} \cdot p_{12}\right)}{\bar{q}_{1}^{2} \bar{q}_{2}^{4} \bar{q}_{12}^{4}}\right] \\
& +\left(\frac{N_{1}^{2}}{\bar{q}_{1}^{4} \bar{S}_{1}}-4 \frac{\left(q_{1} \cdot p_{1}\right)^{2}}{\bar{q}_{1}^{6}}\right)\left[\frac{1}{\bar{q}_{2}^{4}}\right] \\
& +\left(\frac{N_{2}^{2}}{\bar{q}_{2}^{4} \bar{S}_{2}}-4 \frac{\left(q_{2} \cdot p_{2}\right)^{2}}{\bar{q}_{2}^{6}}\right)\left[\frac{1}{\bar{q}_{1}^{4}}\right] \\
& +\left(\frac{N_{12}^{2}}{\bar{q}_{12}^{4} \bar{S}_{12}}-4 \frac{\left(q_{12} \cdot p_{12}\right)^{2}}{\bar{q}_{12}^{6}}\right)\left[\frac{1}{\bar{q}_{1}^{4}}\right]+J_{\mathrm{F}, 2}^{\prime}\left(q_{1}, q_{2}\right) .
\end{aligned}
$$

Then, by rewriting

$$
\frac{m_{i}^{4}}{\bar{D}_{i} \bar{q}_{i}^{4}}=\frac{1}{\bar{D}_{i}}-\frac{1}{\bar{q}_{i}^{2}}-\frac{m_{i}^{2}}{\bar{q}_{i}^{4}}
$$

and shifting all the $\bar{D}_{i}$ and the quadratically divergent integrals, Eq. (8) produces

$$
\begin{aligned}
\int[ & \left.d^{4} q_{1}\right]\left[d^{4} q_{2}\right] \frac{1}{\bar{D}_{1} \bar{D}_{2} \bar{D}_{12}} \\
= & \lim _{\mu \rightarrow 0} \mu_{\mathrm{R}}^{-2 \epsilon} \int d^{n} q_{1} d^{n} q_{2}\left(\frac{1}{\bar{S}_{1} \bar{S}_{2} \bar{S}_{12}}\right. \\
& -\frac{1}{\left(\left(q_{1}+p_{1}\right)^{2}-\mu^{2}\right)\left(\left(q_{2}+p_{2}\right)^{2}-\mu^{2}\right)\left(\left(q_{12}+p_{12}\right)^{2}-\mu^{2}\right)} \\
& -m_{1}^{2}\left[\frac{1}{\bar{q}_{1}^{4} \bar{q}_{2}^{2} \bar{q}_{12}^{2}}\right]-m_{2}^{2}\left[\frac{1}{\bar{q}_{1}^{2} \bar{q}_{2}^{4} \bar{q}_{12}^{2}}\right]-m_{12}^{2}\left[\frac{1}{\bar{q}_{1}^{2} \bar{q}_{2}^{2} \bar{q}_{12}^{4}}\right] \\
& -\left(\frac{1}{\bar{S}_{1}}-\frac{1}{\left(q_{1}+p_{1}\right)^{2}-\mu^{2}}-\frac{m_{1}^{2}}{\bar{q}_{1}^{4}}\right)\left[\frac{1}{\bar{q}_{2}^{4}}\right] \\
& -\left(\frac{1}{\bar{S}_{2}}-\frac{1}{\left(q_{2}+p_{2}\right)^{2}-\mu^{2}}-\frac{m_{2}^{2}}{\bar{q}_{2}^{4}}\right)\left[\frac{1}{\bar{q}_{1}^{4}}\right] \\
& \left.-\left(\frac{1}{\bar{S}_{12}}-\frac{1}{\left(q_{12}+p_{12}\right)^{2}-\mu^{2}}-\frac{m_{12}^{2}}{\bar{q}_{12}^{4}}\right)\left[\frac{1}{\bar{q}_{1}^{4}}\right]\right) .
\end{aligned}
$$

An expansion up to $\mathcal{O}\left(p_{1}^{2}\right), \mathcal{O}\left(p_{2}^{2}\right)$, and $\mathcal{O}\left(p_{1} p_{2}\right)$ of the second line and of the terms

$1 /\left(\left(q_{i}+p_{i}\right)^{2}-\mu^{2}\right)$

in the last three lines produces extra integrands which - by the same argument used at one loop-vanish upon integration. The addition of such terms reconstructs $J_{\mathrm{F}, 2}^{\prime}\left(q_{1}, q_{2}\right)$ as given in Eq. (121), so that

$$
\begin{aligned}
\int\left[d^{4} q_{1}\right]\left[d^{4} q_{2}\right] \frac{1}{\bar{D}_{1} \bar{D}_{2} \bar{D}_{12}} & =\lim _{\mu \rightarrow 0} \int d^{4} q_{1} d^{4} q_{2} J_{\mathrm{F}, 2}^{\prime}\left(q_{1}, q_{2}\right) \\
& =\int\left[d^{4} q_{1}\right]\left[d^{4} q_{2}\right] \frac{1}{\bar{S}_{1} \bar{S}_{2} \bar{S}_{12}} .
\end{aligned}
$$

Finally, taking the derivative with respect to $m_{12}^{2}$ demonstrates the last case.

With more loops the proof follows the same reasoning: the mismatch between the FDR expansion of shifted and unshifted integrands is cured by vanishing integrals obtained by expanding the polynomially divergent integrals in $J_{\mathrm{INF}}\left(q_{1}, \ldots, q_{\ell}\right)$ at the relevant order in $p$, as in Eq. (116).

\section{Appendix B: Feynman rules}

For completeness we list, in Fig. 8, the Feynman rules used in the calculation. $Q_{t}, m_{0}$ and $v$ are the top quark charge, the top bare mass and the vacuum expectation value of the Higgs field, respectively. 
Fig. 8 Feynman rules used in the computation of $H \rightarrow \gamma \gamma$ at $\mathcal{O}\left(\alpha_{S}\right)$

$$
\begin{aligned}
& -\nearrow^{\prime}=-i \frac{m_{0}}{v} \quad \stackrel{p}{\longrightarrow}=-i e Q_{t} \gamma_{\alpha} \quad \stackrel{p}{\longrightarrow}=\frac{i}{\not p-m_{0}} \\
& \overbrace{k, a}^{j}=-i g_{S} \gamma_{\alpha} T_{j k}^{a}
\end{aligned}
$$

\section{Appendix C: A few FDR defining expansions}

We collect here the two-loop FDR defining expansions used throughout the paper. Denominators are defined in Eq. (7) and divergent integrands are written in square brackets.

1. Expansion for $\int\left[d^{4} q_{1}\right]\left[d^{4} q_{2}\right] \frac{q_{1}^{\alpha} q_{1}^{\beta}}{\bar{D}_{1}^{3} \bar{D}_{2} \bar{D}_{12}}$ :

$$
\begin{aligned}
& \frac{q_{1}^{\alpha} q_{1}^{\beta}}{\bar{D}_{1}^{3} \bar{D}_{2} \bar{D}_{12}}=q_{1}^{\alpha} q_{1}^{\beta}\left\{\left[\frac{1}{\bar{q}_{1}^{6} \bar{q}_{2}^{2} \bar{q}_{12}^{2}}\right]\right. \\
& +\left(\frac{1}{\bar{D}_{1}^{3}}-\frac{1}{\bar{q}_{1}^{6}}\right)\left(\left[\frac{1}{\bar{q}_{2}^{4}}\right]-\frac{q_{1}^{2}+2\left(q_{1} \cdot q_{2}\right)}{\bar{q}_{2}^{4} \bar{q}_{12}^{2}}\right) \\
& \left.+\frac{1}{\bar{D}_{1}^{3} \bar{q}_{2}^{2} \bar{D}_{12}}\left(\frac{m_{2}^{2}}{\bar{D}_{2}}+\frac{m_{12}^{2}}{\bar{q}_{12}^{2}}\right)\right\} .
\end{aligned}
$$

2. Expansion for $\int\left[d^{4} q_{1}\right]\left[d^{4} q_{2}\right] \frac{1}{\bar{D}_{1}^{2} \bar{D}_{2} \bar{q}_{12}^{2}}$ :

$$
\begin{aligned}
\frac{1}{\bar{D}_{1}^{2} \bar{D}_{2} \bar{q}_{12}^{2}}= & {\left[\frac{1}{\bar{q}_{1}^{4} \bar{q}_{2}^{2} \bar{q}_{12}^{2}}\right]+\left(\frac{m_{1}^{2}}{\bar{D}_{1} \bar{q}_{1}^{4}}+\frac{m_{1}^{2}}{\bar{D}_{1}^{2} \bar{q}_{1}^{2}}\right) } \\
& \times\left(\left[\frac{1}{\bar{q}_{2}^{4}}\right]-\frac{q_{1}^{2}+2\left(q_{1} \cdot q_{2}\right)}{\bar{q}_{2}^{4} \bar{q}_{12}^{2}}\right) \\
& +\frac{m_{2}^{2}}{\bar{D}_{1}^{2}\left(\bar{D}_{2} \bar{q}_{2}^{2}\right) \bar{q}_{12}^{2}} .
\end{aligned}
$$

3. Expansion for $\int\left[d^{4} q_{1}\right]\left[d^{4} q_{2}\right] \frac{q_{1}^{\alpha} q_{1}^{\beta}}{\bar{D}_{1}^{2} \bar{D}_{2}^{2} \bar{q}_{12}^{2}}$ :

$$
\begin{aligned}
\frac{q_{1}^{\alpha} q_{1}^{\beta}}{\bar{D}_{1}^{2} \bar{D}_{2}^{2} \bar{q}_{12}^{2}}= & q_{1}^{\alpha} q_{1}^{\beta}\left\{\left[\frac{1}{\bar{q}_{1}^{4} \bar{q}_{2}^{4} \bar{q}_{12}^{2}}\right]+\left(\frac{m_{2}^{2}}{\bar{D}_{2} \bar{q}_{2}^{4}}+\frac{m_{2}^{2}}{\bar{D}_{2}^{2} \bar{q}_{2}^{2}}\right)\right. \\
& \times\left(\left[\frac{1}{\bar{q}_{1}^{6}}\right]-\frac{q_{2}^{2}+2\left(q_{1} \cdot q_{2}\right)}{\bar{q}_{1}^{6} \bar{q}_{12}^{2}}\right) \\
& \left.+\left(\frac{1}{\bar{D}_{1}^{2}}-\frac{1}{\bar{q}_{1}^{4}}\right) \frac{1}{\bar{D}_{2}^{2} \bar{q}_{12}^{2}}\right\} .
\end{aligned}
$$

4. Expansion for $\int\left[d^{4} q_{1}\right]\left[d^{4} q_{2}\right] \frac{q_{12}^{\alpha} q_{12}^{\beta}}{\bar{D}_{1}^{2} \bar{D}_{2}^{2} \bar{q}_{12}^{2}}$ :

$$
\begin{aligned}
\frac{q_{12}^{\alpha} q_{12}^{\beta}}{\bar{D}_{1}^{2} \bar{D}_{2}^{2} \bar{q}_{12}^{2}}= & q_{12}^{\alpha} q_{12}^{\beta}\left\{\left[\frac{1}{\bar{q}_{1}^{4} \bar{q}_{2}^{4} \bar{q}_{12}^{2}}\right]+\left[\frac{1}{\bar{q}_{12}^{6}}\right]\left(\left(\frac{m_{1}^{2}}{\bar{D}_{1} \bar{q}_{1}^{4}}\right.\right.\right. \\
& \left.\left.+\frac{m_{1}^{2}}{\bar{D}_{1}^{2} \bar{q}_{1}^{2}}\right)+\left(\frac{m_{2}^{2}}{\bar{D}_{2} \bar{q}_{2}^{4}}+\frac{m_{2}^{2}}{\bar{D}_{2}^{2} \bar{q}_{2}^{2}}\right)\right) \\
& +\frac{1}{\bar{q}_{12}^{2}}\left(\left(\frac{1}{\bar{D}_{1}^{2}}-\frac{1}{\bar{q}_{1}^{4}}\right)\left(\frac{1}{\bar{D}_{2}^{2}}-\frac{1}{\bar{q}_{2}^{4}}\right)\right. \\
& +\left(\frac{1}{\bar{q}_{1}^{4}}-\frac{1}{\bar{q}_{12}^{4}}\right)\left(\frac{1}{\bar{D}_{2}^{2}}-\frac{1}{\bar{q}_{2}^{4}}\right) \\
& \left.\left.+\left(\frac{1}{\bar{q}_{2}^{4}}-\frac{1}{\bar{q}_{12}^{4}}\right)\left(\frac{1}{\bar{D}_{1}^{2}}-\frac{1}{\bar{q}_{1}^{4}}\right)\right)\right\} .
\end{aligned}
$$

\section{Appendix D: The basic two-loop scalar integral}

In this appendix, we demonstrate that the basic two-loop scalar integral in Eq. (95) reads

$$
\begin{aligned}
& {\left[2 m_{1}\left|m_{2}\right| 0\right] \equiv \int\left[d^{4} q_{1}\right]\left[d^{4} q_{2}\right] \frac{1}{\bar{D}_{1}^{2} \bar{D}_{2} \bar{q}_{12}^{2}}} \\
& =\pi^{4}\left\{f-\mathrm{Li}_{2}\left(1-\frac{m_{2}^{2}}{m_{1}^{2}}\right)-\frac{1}{2} \ln ^{2} \frac{\mu_{\mathrm{R}}^{2}}{m_{1}^{2}}-\ln \frac{\mu_{\mathrm{R}}^{2}}{m_{1}^{2}}\right\}
\end{aligned}
$$

with

$f=\frac{i}{\sqrt{3}}\left(\operatorname{Li}_{2}\left(e^{i \frac{\pi}{3}}\right)-\operatorname{Li}_{2}\left(e^{-i \frac{\pi}{3}}\right)\right)$.

A direct integration of the finite part of its FDR defining expansion-Eq. (126)—gives

$\left[2 m_{1}\left|m_{2}\right| 0\right]=m_{2}^{2} I_{2}\left(m_{1}, m_{2}\right)-m_{1}^{2} I_{1}\left(m_{1}\right)$, 
where

$$
\begin{aligned}
I_{2}\left(m_{1}, m_{2}\right)= & \lim _{\mu \rightarrow 0} \int d^{4} q_{1} d^{4} q_{2} \frac{1}{\bar{D}_{1}^{2}\left(\bar{D}_{2} \bar{q}_{2}^{2}\right) \bar{q}_{12}^{2}} \text { and } \\
I_{1}\left(m_{1}\right)= & \lim _{\mu \rightarrow 0} \int d^{4} q_{1} d^{4} q_{2} \frac{q_{1}^{2}+2\left(q_{1} \cdot q_{2}\right)}{\bar{q}_{2}^{4} \bar{q}_{12}^{2}} \\
& \times\left(\frac{1}{\bar{D}_{1} \bar{q}_{1}^{4}}+\frac{1}{\bar{D}_{1}^{2} \bar{q}_{1}^{2}}\right) .
\end{aligned}
$$

By power counting — due to the presence of $1 / q_{i}^{4}$ terms a logarithmic dependence on $\mu$ is expected in $I_{1}\left(m_{1}\right)$, while $\mu$ can be immediately set to zero in $I_{2}\left(m_{1}, m_{2}\right)$. A natural split is then obtained in FDR: $I_{2}\left(m_{1}, m_{2}\right)$ only depends on

$r_{12}=\frac{m_{1}^{2}}{m_{2}^{2}}$,

and $I_{1}\left(m_{1}\right)$ on

$\rho_{1}=\frac{\mu^{2}}{m_{1}^{2}}$,

so no difficult integral containing both ratios needs to be computed. A simple Feynman parametrization produces

$$
\begin{aligned}
I_{2}\left(m_{1}, m_{2}\right) & =\int_{0}^{1} d z \int d^{4} q_{1} d^{4} q_{2} \frac{1}{D_{1}^{2}\left(q_{2}^{2}-m_{2}^{2} z\right)^{2} q_{12}^{2}} \\
& =\frac{\pi^{4}}{m_{2}^{2}} \int_{0}^{1} d z \int_{0}^{1} d x \int_{0}^{1} d y \frac{y}{x y z+r_{12}(1-y)} \\
& =\frac{\pi^{4}}{m_{2}^{2}}\left\{\frac{\pi^{2}}{6}-\operatorname{Li}_{2}\left(\frac{r_{12}-1}{r_{12}}\right)\right\}
\end{aligned}
$$

and

$$
\begin{aligned}
I_{1}\left(m_{1}\right)= & 2 \lim _{\mu \rightarrow 0} \int_{0}^{1} d z \int d^{4} q_{1} d^{4} q_{2} \frac{q_{1}^{2}+2\left(q_{1} \cdot q_{2}\right)}{\left(\bar{q}_{1}^{2}-m_{1}^{2} z\right)^{3} \bar{q}_{2}^{4} \bar{q}_{12}^{2}} \\
= & \frac{2 \pi^{4}}{m_{1}^{2}} \lim _{\mu \rightarrow 0} \int_{0}^{1} d z \int_{0}^{1} d x \int_{0}^{1} d y \\
& \times \frac{(2 x-1) y^{2} x}{\left(z+\rho_{1}\right) x(1-x) y+\rho_{1}(1-y)} \\
= & \frac{\pi^{4}}{m_{1}^{2}}\left\{\frac{\pi^{2}}{6}-f+\frac{1}{2} \ln ^{2} \rho_{1}+\ln \rho_{1}\right\},
\end{aligned}
$$

from which Eq. (129) follows. The same result can be derived as a finite combination of divergent integrals. From Eq. (126), by using DR,

$$
\begin{aligned}
{\left[2 m_{1}\left|m_{2}\right| 0\right]=} & \mu_{\mathrm{R}}^{-2 \epsilon} \int d^{n} q_{1} d^{n} q_{2} \frac{1}{D_{1}^{2} D_{2} q_{12}^{2}} \\
& -\lim _{\mu \rightarrow 0} \mu_{\mathrm{R}}^{-2 \epsilon} \int d^{n} q_{1} d^{n} q_{2}\left[\frac{1}{\bar{q}_{1}^{4} \bar{q}_{2}^{2} \bar{q}_{12}^{2}}\right] \\
& -\lim _{\mu \rightarrow 0} \mu_{\mathrm{R}}^{-2 \epsilon} \int d^{n} q_{2}\left[\frac{1}{\bar{q}_{2}^{4}}\right] \int d^{n} q_{1} \\
& \times\left(\frac{m_{1}^{2}}{\bar{D}_{1} \bar{q}_{1}^{4}}+\frac{m_{1}^{2}}{\bar{D}_{1}^{2} \bar{q}_{1}^{2}}\right)
\end{aligned}
$$

The relevant DR integrals can be found in the appendix of [51].

\section{References}

1. J. Blumlein, PoS RADCOR2011, 048 (2011)

2. F. Simon, hep-ex/1401.6302 (2014)

3. G. Passarino, M. Veltman, Nucl. Phys. B 160, 151 (1979). doi:10. 1016/0550-3213(79)90234-7

4. S. Frixione, Z. Kunszt, A. Signer, Nucl. Phys. B 467, 399 (1996). doi:10.1016/0550-3213(96)00110-1

5. S. Catani, M. Seymour, Nucl. Phys. B 485, 291 (1997). doi:10. 1016/S0550-3213(96)00589-5

6. D.A. Kosower, Phys. Rev. D 57, 5410 (1998). doi:10.1103/ PhysRevD.57.5410

7. J.M. Campbell, M. Cullen, E.N. Glover, Eur. Phys. J. C 9, 245 (1999). doi:10.1007/s100529900034

8. S. Catani, S. Dittmaier, M.H. Seymour, Z. Trocsanyi, Nucl. Phys. B 627, 189 (2002). doi:10.1016/S0550-3213(02)00098-6

9. Z. Nagy, D.E. Soper, JHEP 0309, 055 (2003)

10. Z. Bern, L.J. Dixon, D.C. Dunbar, D.A. Kosower, Nucl. Phys. B 435, 59 (1995). doi:10.1016/0550-3213(94)00488-Z

11. R. Britto, F. Cachazo, B. Feng, Nucl. Phys. B 725, 275 (2005). doi:10.1016/j.nuclphysb.2005.07.014

12. G. Ossola, C.G. Papadopoulos, R. Pittau, Nucl. Phys. B 763, 147 (2007). doi:10.1016/j.nuclphysb.2006.11.012

13. D. Forde, Phys. Rev. D 75, 125019 (2007). doi:10.1103/PhysRevD. 75.125019

14. C. Berger, Z. Bern, L. Dixon, F. Febres, Cordero, D. Forde, et al. Phys. Rev. D 78, 036003 (2008). doi:10.1103/PhysRevD.78. 036003

15. R.K. Ellis, K. Melnikov, G. Zanderighi, JHEP 0904, 077 (2009). doi:10.1088/1126-6708/2009/04/077

16. G. Passarino, Nucl. Phys. B 619, 257 (2001). doi:10.1016/ S0550-3213(01)00528-4

17. P. Mastrolia, G. Ossola, JHEP 1111, 014 (2011). doi:10.1007/ JHEP11(2011)014

18. P. Mastrolia, E. Mirabella, G. Ossola, T. Peraro, Phys. Lett. B 718 , 173 (2012). doi:10.1016/j.physletb.2012.09.053

19. R.H. Kleiss, I. Malamos, C.G. Papadopoulos, R. Verheyen, JHEP 1212, 038 (2012). doi:10.1007/JHEP12(2012)038

20. S. Badger, H. Frellesvig, Y. Zhang, JHEP 1204, 055 (2012). doi:10. 1007/JHEP04(2012)055

21. H. Johansson, D.A. Kosower, K.J. Larsen, Phys. Rev. D 87, 025030 (2013). doi:10.1103/PhysRevD.87.025030

22. A. Gehrmann-De Ridder, T. Gehrmann, E.N. Glover, JHEP 0509, 056 (2005). doi:10.1088/1126-6708/2005/09/056

23. J. Currie, A. Gehrmann-De Ridder, E. Glover, J. Pires, JHEP 1401, 110 (2014). doi:10.1007/JHEP01(2014)110 
24. T. Binoth, G. Heinrich, Nucl. Phys. B 585, 741 (2000). doi:10. 1016/S0550-3213(00)00429-6

25. C. Anastasiou, K. Melnikov, F. Petriello, Phys. Rev. D 69, 076010 (2004). doi:10.1103/PhysRevD.69.076010

26. T. Binoth, G. Heinrich, Nucl. Phys. B 693, 134 (2004). doi:10. 1016/j.nuclphysb.2004.06.005

27. M. Czakon, Phys. Lett. B 693, 259 (2010). doi:10.1016/j.physletb. 2010.08 .036

28. S. Catani, M. Grazzini, Phys. Rev. Lett. 98, 222002 (2007). doi:10. 1103/PhysRevLett.98.222002

29. G. 't Hooft, M. Veltman, Nucl. Phys. B 44, 189 (1972). doi:10. 1016/0550-3213(72)90279-9

30. R. Pittau, JHEP 1211, 151 (2012). doi:10.1007/JHEP11(2012)151

31. D.Z. Freedman, K. Johnson, J.I. Latorre, Nucl. Phys. B 371, 353 (1992). doi:10.1016/0550-3213(92)90240-C

32. F. del Aguila, A. Culatti, R. Munoz-Tapia, M. Perez-Victoria, Phys. Lett. B 419, 263 (1998). doi:10.1016/S0370-2693(97)01279-3

33. F. del Aguila, A. Culatti, R. Munoz, Tapia. M. Perez-Victoria. Nucl. Phys. B 537, 561 (1999). doi:10.1016/S0550-3213(98)00645-2

34. O. Battistel, A. Mota, M. Nemes, Mod. Phys. Lett. A 13, 1597 (1998). doi:10.1142/S0217732398001686

35. G. Cynolter, E. Lendvai, Central. Eur. J. Phys. 9, 1237 (2011). doi:10.2478/s11534-011-0039-y

36. A. Cherchiglia, M. Sampaio, M. Nemes, Int. J. Mod. Phys. A 26, 2591 (2011). doi:10.1142/S0217751X11053419

37. R. Pittau, Eur. Phys. J. C 74, 2686 (2014). doi:10.1140/epjc/ s10052-013-2686-1

38. A.M. Donati, R. Pittau, JHEP 1304, 167 (2013). doi:10.1007/ JHEP04(2013) 167
39. R. Pittau, hep-ph/1305.0419 (2013)

40. G.F. Sterman, An Introduction to quantum field theory (Cambridge University Press, 1994)

41. J.C. Collins, Renormalization (Cambridge University Press, 1984)

42. M. Veltman, in Proceedings of the VI International Symposium on Electron and Proton Interaction at High Energies (1973), pp. $429-447$

43. G. 't Hooft, M. Veltman, NATO Adv. Study Inst. Ser. B Phys. 4, 177 (1974)

44. Z. Bern, A. De Freitas, L.J. Dixon, H. Wong, Phys. Rev. D 66, 085002 (2002). doi:10.1103/PhysRevD.66.085002

45. W. Siegel, Phys. Lett. B 84, 193 (1979). doi:10.1016/ 0370-2693(79)90282-X

46. Z. Bern, A. Morgan, Nucl. Phys. B 467, 479 (1996). doi:10.1016/ 0550-3213(96)00078-8

47. G. Cullen, N. Greiner, G. Heinrich, G. Luisoni, P. Mastrolia et al., Eur. Phys. J. C72, 1889 (2012). doi:10.1140/epjc/ s10052-012-1889-1

48. G. Leibbrandt, Rev. Modern Phys. 47(4), 849 (1975)

49. W. Giele, E.N. Glover, D.A. Kosower, Nucl. Phys. B 403, 633 (1993). doi:10.1016/0550-3213(93)90365-V

50. A. Djouadi, M. Spira, J. van der Bij, P. Zerwas, Phys. Lett. B 257, 187 (1991). doi:10.1016/0370-2693(91)90879-U

51. J. van der Bij, M. Veltman, Nucl. Phys. B 231, 205 (1984). doi:10. 1016/0550-3213(84)90284-0 